\title{
A cytoplasmic protein kinase in Chlamydomonas couples engagement of ciliary receptors to rapid cellular responses
}

Mayanka Awasthi ${ }^{1}$, Peeyush Ranjan ${ }^{1}$, Simon Kelterborn ${ }^{2,3}$, Peter Hegemann² and William J. Snell ${ }^{1 *}$

\begin{abstract}
${ }^{1}$ Department of Cell Biology and Molecular Genetics, University of Maryland, College Park, MD
\end{abstract}
${ }^{2}$ Experimental Biophysics, Institute for Biology, Humboldt-Universität zu Berlin, Berlin, Germany.

${ }^{3}$ Charité - Universitätsmedizin Berlin, Institute of Translational Physiology, Berlin, Germany

*Correspondence: wsnell1@umd.edu

\section{Abstract}

The principal function of the primary cilium is to convert cues from the extracellular milieu into changes in cyclic nucleotide concentration and cytoplasmic responses, but fundamental questions remain about the mechanisms of transmission of cilium-tocytoplasm signals. During fertilization in Chlamydomonas reinhardtii, ciliary adhesion between plus and minus gametes triggers an immediate $\sim 10$-fold increase in cellular cAMP and activation for cell fusion. Here, we identify Gamete- $\underline{\text { Specific }}$ Protein $\underline{\text { Kinase }}$ (GSPK) as an essential link between cilary receptor engagement and gamete activation. The ciiary adhesion-induced increase in cAMP and cell fusion are severely impaired in gspk mutants but fusion is rescued by a cell-permeable form of cAMP, indicating that GSPK functions upstream of the CAMP increase. GSPK is cytoplasmic, and, remarkably, the entire cellular complement is phosphorylated in less than 60 seconds after ciliary contact. Thus, a cytoplasmic protein kinase rapidly converts a ciliary membrane cue into a global cellular response.

\section{Introduction}

The primary cilium is a spatially distinct cellular compartment specialized for receipt of extracellular signals. Its membrane houses multiple receptors essential for development and homeostasis ${ }^{1-3}$, including G-protein coupled receptors (GPCRs) responsible for vision ${ }^{4}$, olfaction ${ }^{5,6}$, growth factor receptor-activated pathways ${ }^{7}$, regulation of insulin and glucagon secretion in pancreatic islet cells ${ }^{8}$, and the sonic hedgehog $(\mathrm{Hh})$ developmental pathway ${ }^{9}$. And, cilia participate in regulation of 
epithelial cell proliferation in the liver, gall bladder, and kidney ${ }^{3,10,11}$. The ciliary compartment is unique in its combined functional and physical separation from the cell proper and is surrounded, not by cytoplasm, as are intracellular membrane-bounded organelles, but by the extracellular milieu. Moreover, the membrane at the connection between the cilium and the cell is packed with membrane protein complexes tightly linked to the dense array of underlying doublet microtubules of the ciliary axoneme ${ }^{12-}$ ${ }^{14}$, thereby providing a regulatable barrier to membrane protein movement between the two domains.

Although the multiple signaling pathways initiated in the cilium vary widely across cell types in their mechanisms of activation and in their downstream outcomes, almost all are linked by their common function in regulating the concentrations of cyclic nucleotides, primarily the second messenger, cAMP. Increases in ciliary cAMP upon odorant binding by cilia of olfactory epithelial cells alter the activity of cyclic nucleotidegated ion channels within the cilia, thereby inducing changes in membrane potential that are transmitted through the cell body and axons to generate a sense of smell ${ }^{15}$. Changes in ciliary cAMP upon activation of the cilium-based sonic hedgehog $(\mathrm{Hh})$ and other GPCR-regulated pathways alter the activities of cAMP-dependent protein kinases and EPACs through multiple, complex mechanisms that lead to changes in protein secretion and gene expression ${ }^{16-18}$. During kidney tubule development, the increased cAMP that is a consequence of mutation of ciliary proteins polycystin I, polycystin 2, fibrocystin, and others leads to polycystic kidney disease (PKD) ${ }^{3,11}$. In spite of the importance of cilium-initiated, cAMP-dependent signaling pathways, however, fundamental questions remain about the mechanisms that couple receptor activity at the ciliary membrane to downstream responses in the cell.

In many unicellular organisms, including parasitic protozoans, ciliated protozoans, and green algae, cues received at cilia trigger changes in ciliary cAMP and cellular responses, and thus this ciliary signaling strategy is ancient ${ }^{19-23}$. During sexual reproduction in the bi-ciliated, unicellular green alga Chlamydomonas reinhardtii, interactions between the cilia of gametes of opposite mating types trigger a rapid, 10fold increase in intracellular cAMP that activates the gametes for cell-cell fusion ${ }^{22-26}$. 
of plus gametes and adhesion receptor SAD1 on the cilia of minus gametes ${ }^{27,28}$. During this flirtation with multicellularity by a unicellular organism, the signaling pathway activated by SAG1-SAD1 interactions exhibits many of the hallmarks of pathways activated by receptor-ligand interactions in animal cells, including changes in the phosphorylation states of proteins and changes in protein location ${ }^{23-26,29,30}$. Chlamydomonas ciliary signaling has proved useful for understanding fundamental signaling properties of cilia. Studies over 35 years ago demonstrated the existence of a functional barrier between the Chlamydomonas plasma membrane and the ciliary membrane ${ }^{31,32}$. Related studies showed that, contrary to then emerging models, regulated movement of membrane proteins into the cilia does not require intraflagellar transport (IFT) $)^{33-35}$.

Studies with cilia isolated separately from naive (resting or unmixed) plus and minus gametes and from gametes soon after mixing have shown that within seconds after cilia adhere to each other, a cGMP-dependent protein kinase (PKG) becomes phosphorylated $^{36,37}$. Although the protein kinase that phosphorylates the PKG and the substrates for the PKG are unknown, experimentally reducing expression of the PKG impairs gamete fusion ${ }^{37}$. Related studies have also shown that cilia isolated from Chlamydomonas gametes possess an adenylyl cyclase activity that is regulated by phosphorylation and dephosphorylation ${ }^{25,26}$. The in vitro activity of this as yet unidentified ciliary adenylyl cyclase is increased $\sim 2$-fold after plus and minus gametes are mixed together ${ }^{24}$, and even when isolated cilia are mixed together ${ }^{26}$. Moreover, cell bodies possess an adenylyl cyclase activity detected by in vitro assays, that is also increased $\sim 2$-fold by ciliary adhesion. The adhesion-induced increase in the ciliary adenylyl cyclase activity occurs within 1 minute after gametes are mixed together, and the increase in the activity of the cell body adenylyl cyclase occurs within 2 minutes. Although, one model is that the cAMP formed in the cilia activates the adenylyl cyclase in the cell body ${ }^{24}$, the relationship between the ciliary adenylyl cyclase activity and gamete activation is unknown.

The primary cellular responses to the ciliary adhesion-triggered increase in cellular cAMP occur in the cell body within $\sim 2$ minutes after gametes are mixed together. The gametes release their cell walls ${ }^{38}$, mobilize pools of SAG1 and SAD1 from the plasma 
membrane onto the ciliary membrane in a positive feedback mechanism that sustains and enhances adhesion, ${ }^{30,34,39-41}$ and erect fusogenic membrane protuberances - mating structures ${ }^{42}$ - - as the gametes prepare for fusion to form a quadri-ciliated zygote (Fig. 1a). These cellular responses to ciliary adhesion (with the exception of gamete fusion) can be mimicked experimentally by incubation of gametes of a single mating type in a buffer containing a cell-permeable analogue of CAMP, dibutyryl cAMP (db-cAMP) ${ }^{22}$. As in other systems, however, the molecules and mechanisms that couple ciliary receptor engagement in Chlamydomonas gametes to CAMP-dependent cellular responses in the cytoplasm remain poorly understood.

Here, we report identification of a gamete-specific protein kinase, Gamete Specific Protein Kinase (GSPK), that is essential for this cilium-based signaling pathway. GSPK has sequence homology to mixed lineage protein kinases of animal cells and cell fractionation shows that it is a cell body protein. GSPK is basally phosphorylated in naive gametes and undergoes further phosphorylation within 1 minute after plus and minus gametes are mixed together. Studies with gspk mutants indicate that the protein is essential for gamete fusion and functions downstream of ciliary adhesion and the adhesion-induced phosphorylation of ciliary PKG. Cell body responses to ciliary adhesion are strongly impaired in gspk gametes, and the rapid cell-cell fusion that typifies wild type gametes fails to occur in the mutants. Experiments showing rescue of fusion in mutant gametes by db-cAMP indicate that the downstream gamete activation machinery is intact in the mutants. Importantly, assays of cellular cAMP indicate that ciliary adhesion in the mutant gametes fails to induce the large increase in cAMP that typically accompanies ciliary adhesion. Our results indicate that GSPK is a cytoplasmic protein that rapidly detects ciliary adhesion and couples engagement of ciliary adhesion receptors to CAMP-dependent responses in the cytoplasm required for cell-cell fusion.

\section{Results}

Identification of a gamete-specific protein kinase essential for fertilization in Chlamydomonas.

To identify protein kinases with a potential role in ciliary signaling during fertilization, we tested for a cell-cell fusion phenotype in several minus mating-type strains from the Chlamydomonas CLiP mutant library that were annotated to contain mutations in 
protein kinase genes and that exhibited gamete-specific expression profiles ${ }^{43}$ (Fig. 1b). Of 9 strains examined, gametes prepared from strain LMJ.RY0402.138658, which was annotated to have an insertion of the antibiotic resistance cassette APHVIII in gene Cre02.g104450 (whose encoded protein is GSPK), was strongly impaired in gamete fusion when mixed with wild type (WT) plus gametes (Fig. 1b, c). PCR analyses using gene-specific and cassette-specific sets of primers confirmed APHVIII cassette insertion into exon 3 in strain LMJ.RY0402.138658 (gspk-1) (Fig. 1d and Supplementary Table 1) and at the predicted insertion sites in two other independent

143 CLiP library strains annotated to have insertions in Cre02.g104450, 144 LMJ.RY0402.097798 (gspk-2; predicted insertion in exon 8) and LMJ.RY0402.039382 (gspk-3; predicted insertion in the 3'UTR) (Supplementary Table 1 and Supplementary Fig. 1). Determination of the percent of gametes that fused after mixing with WT plus gametes confirmed that GSPK indeed was essential for the rapid fusion that typifies WT gametes. Nearly $70 \%$ of the cells in mixtures of WT minus gametes and WT plus gametes had fused to form quadri-ciliated cells (zygotes) within 10 minutes after mixing (Fig. 1c), whereas, fusion was less than $1 \%$ for each of the three minus mutant strains (Fig. 1C). The percent fusion in the mutants increased slightly by 60 minutes '(to 15-18\%) (Fig. 1c).

Analysis of progeny from zygotes produced by crossing (Supplementary Fig. 2) gspk1 minus gametes with WT plus gametes showed that both minus gametes and plus gametes bearing the gspk-1 allele were defective in fusion when mixed with $W T$ gametes of the opposite mating type (Fig. 1e). The identical phenotype was also found in a separate Cre02.g104450 mutant strain $\Delta g s p k-d 2$ generated in plus cells by use of CRISPR methods (Fig. 1e; Supplementary Fig. 3). Thus, the gspk mutation segregated with the mutant phenotype, and gspk gametes of both mating types exhibited the fusion phenotype.

163 Examination of sequence alignments showed that GSPK possessed the canonical protein kinase sub-domains of members of the protein kinase superfamily and was most closely related to mixed lineage protein kinases (Fig. 1f). GSPK contains $\sim 151$ residues between subdomains IV and $\mathrm{V}$ absent in most other protein kinases that

167 could be a potential regulatory motif. Use of NMT - The MYR Predictor 
(https://mendel.imp.ac.at/myristate/SUPLpredictor.htm) to predict N-myristoylation sites indicated that the glycine at position 2 in GSPK (MGAVLSCCGEGTIGASHG) is a potential myristoylation site.

172 To investigate the cellular properties of GSPK, we introduced into gspk cells a 173 transgene encoding an epitope-tagged form of GSPK, GSPK-HA, driven by the endogenous promoter. Immunoblotting of gspk and gspk/GSPK-HA minus gametes with anti-HA antbodies showed a tagged protein of the expected size, $\sim 70 \mathrm{kDa}$, only in the cells bearing the transgene (Fig. 2a). Consistent with the analysis above indicating that mutation of GSPK was responsible for the fusion phenotype, introduction of the GSPK-HA transgene rescued fusion (Fig. 2a). Furthermore, and consistent with the transcriptome evidence (Fig. 1b), GSPK-HA was expressed only in gametes and not vegetative cells, and activation of the gametes by incubation in db-cAMP buffer for 1 hour brought about a substantial reduction in GSPK-HA protein levels (Fig. 2b).

The entire cellular complement of GSPK is in the cytoplasm and is phosphorylated within 1 minute after ciliary receptor engagement.

We used SDS-PAGE and immunoblotting to assess the phosphorylation state of GSPK-HA in naive plus gametes and in plus gametes undergoing ciliary adhesion at increasing times after mixing with hap2 minus gametes. As shown in Fig. 2c, incubation of lysates of naive GSPK-HA gametes with the protein de-phosphorylating enzyme, I-phosphatase, led to a shift in migration of GSPK-HA compared to the nontreated sample or compared to a sample incubated with the phosphatase and a phosphatase inhibitor. These results indicated that GSPK was basally phosphorylated in naive gametes.

Similar analysis showed that ciliary adhesion induced a further increase in GSPK-HA phosphorylation. Upon mixing the GSPK-HA(+) gametes with hap2 minus gametes, which are defective in gamete fusion because they fail to express the gamete fusogen, HAP2 ${ }^{44}$, the basally phosphorylated GSPK-HA underwent a shift in migration (Fig. $2 \mathrm{~d}$ ). 
shift was detectable within 1 minute after mixing. Consistent with the shift being a consequence of phosphorylation, all of the GSPK-HA was shifted to the unphosphorylated form upon incubation of the lysates with I-phosphatase (Fig. 2e).

Given that all of the GSPK underwent the ciliary adhesion-induced rapid phosphorylation, it seemed likely that the protein itself would be localized in the organelles. Analysis by immunoblotting, however, of naive whole cells, cell bodies, and cilia indicated that GSPK was present in cell bodies, with little if any detectable in the cilia (Fig. 2f). Moreover, even though all other cell body events that occur during gamete interactions can be induced in gamete of a single mating type by incubation in db-cAMP ${ }^{32,41}$, phosphorylation of GSPK-HA was not induced by db-cAMP (Fig. 2g). (Cell wall loss was over $80 \%$ at 10 minutes in these samples). Thus, interactions between SAG1 and SAD1 at the surface of the cilia were rapidly transduced into phosphorylation of GSPK in the cell body, but GSPK phosphorylation was upstream of the increase in CAMP that drives gamete activation.

\section{The earliest biochemically detectable response in cilia to ciliary adhesion,} phosphorylation of a cGMP-dependent protein kinase, does not require GSPK.

To examine the cellular function of GSPK, we further investigated the phenotype of gspk mutants. Vegetative cells of both plus and minus gspk strains were indistinguishable from wild-type vegetative cells in size, appearance, motility, and growth. Moreover, all of the gspk mutant strains underwent normal gametogenesis to form gametes that were indistinguishable from the wild-type gametes in morphology and motility (not shown). Similarly, microscopic examination (Fig. 3ai) and a quantitative assay for ciliary adhesion (Fig. 3aii) showed that the gspk minus gametes underwent initial ciliary adhesion with $W T$ plus gametes to nearly the same extent as did fusion-defective hap2 minus gametes with WT plus gametes. gspk plus gametes were similarly competent for ciiary adhesion with WT minus gametes (not shown). Thus, GSPK functioned downstream of SAG1-SAD1-dependent ciliary adhesion. 
We tested whether the earliest experimentally detectable consequence of ciliary

234 adhesion, phosphorylation of ciliary PKG (Fig. 3b), was intact in the gspk mutants.

235 Cilia isolated from WT plus and minus gametes that had been mixed together for 3

236 minutes and from gspk mutant plus and minus gametes mixed for the same time were

237 assessed for tyrosine phosphorylation of PKG by an in vitro assay and immunoblotting

238 with anti-phosphotyrosine antibodies ${ }^{36}$. As shown in Fig. 3c, phosphorylation of the

$239105 \mathrm{kDa}$ PKG was at very low levels in assays of cilia isolated from naive plus gametes

240 and from naive minus WT gametes and in assays of separately isolated cilia from

241 naive plus and minus gspk gametes. On the other hand, cilia isolated from the mixed

242 WT plus and minus gametes and from the mixed gspk plus and minus gametes

243 undergoing ciliary adhesion showed robust phosphorylation of PKG in the assays.

244 Thus, the earliest biochemical response within cilia to SAG1-SAD1 interactions was

245 independent of GSPK.

Ciliary adhesion by gspk gametes fails to induce the cell body responses required for gamete fusion.

Given that the block to fusion in the gspk mutants was downstream of initial ciliary events, we used bioassays to determine whether gspk gametes underwent the typical cell body responses to ciliary adhesion (Fig. 3b). Our wall loss assay indicated that cell wall release was severely impaired in the gspk gametes (Fig. 4a). Whereas nearly $70 \%$ of the cells in samples of adhering WT plus gametes mixed with hap2 minus gametes had lost their walls at 10 minutes after mixing, fewer than $20 \%$ of the mixed gspk gametes had lost their walls. Similary, mating structure activation, as measured by the appearance of the actin-filled microvillous-like fertilization tubules in plus gametes was substantially reduced in the gspk gametes (Fig. 4b). 30 minutes after mixing equal numbers of WT plus gametes with hap 2 minus gametes, nearly $45 \%$ of the cells in the mixture possessed actin-staining mating structures (which meant that $\sim 90 \%$ of the plus gametes had formed mating structure), but fewer than $3 \%$ of the gspk plus gametes had formed the structures (Fig. 4b and Supplementary Fig. 4).

264 We also investigated the ability of gspk plus gametes to recruit SAG1 from the cell body to the cilia, a response to ciliary signaling that maintains and enhances ciliary adhesion $^{45}$. We obtained gspk plus cells bearing HA-tagged SAG1 from a cross 
between gspk(-) gametes with SAG1-HA(+) gametes (Supplementary Fig. 2). gspk/SAG1-HA(+) gametes were mixed with fusion-defective hap2(-)gametes, and at 0,10 , and 45 minutes after mixing, samples were harvested, fractionated, and whole cells, cell bodies, and cilia were analyzed by anti-HA immunoblotting. Samples from a mixture of adhering SAG1-HA and hap2 gametes served as controls. As expected, immunoblots of equal amounts of protein showed that SAG1-HA was present at low levels in cilia compared to the cell bodies in the 0-time samples of both the WT and gspk(+) gametes (Fig. 4c). Moreover, at 10 minutes after mixing, SAG1-HA had been recruited into both the $W T$ and the gspk cilia. On other hand, at 45 minutes after mixing the amount of SAG1-HA had increased in the cilia of the WT plus gametes, but the amount of SAG1-HA in the cilia of the gspk gametes had decreased (Fig. 4c). Thus, although it was dispensable for the initial, adhesion-induced recruitment of SAG1-HA to cilia, GSPK was required to sustain SAG1 recruitment.

Ciliary adhesion is dynamic, and sites of adhesion are constantly being formed and broken along the lengths of the cilia as the organelles release membrane vesicles (ciliary ectosomes) enriched in SAG1 and SAD140. Maintenance of ciliary adhesion, thus, depends on recruitment of SAG1 from the inactive pool on the surface of the cell body membrane. Indeed, consistent with the decreased amount of SAG1-HA in the cilia of the gspk gametes at 30 minutes after mixing (Fig. 4c), examination by phasecontrast microscopy of the 30 minute samples of the gspk/SAG1-HA plus gametes mixed with the fusion-defective hap2 minus gametes samples indicated that many had become single cells. And, by 45 minutes few if any were in clusters. On the other hand, in the control sample of WT plus gametes mixed with fusion-defective hap2 minus gametes, the cells continued to adhere to each other and the clusters had grown even larger (Fig. 4d). Thus, both biochemical and functional evidence indicated that sustained recruitment of adhesion molecules during ciliary adhesion required GSPK.

Taken together, the results above suggested that GSPK functioned upstream of all of the cell body events in the gamete activation pathway. Consistent with this interpretation, when db-cAMP was added to gspk gametes, they underwent cell wall loss and mating structure activation similarly to WT gametes (Fig. 4a, b). Furthermore, in the presence of db-cAMP, gspk plus and minus gametes were fully capable of 
undergoing cell-cell fusion (Fig. 4e), suggesting that GSPK was a positive regulator of CAMP. Indeed, assays for cellular cAMP showed that whereas the levels of this seond messenger increased nearly 10-fold when WT plus and minus gametes were mixed together, the cAMP increase was transient and less than 2-fold when plus and minus gspk mutants were mixed together (Fig. 4f). Thus, the rapid increase in cellular cAMP triggered by ciliary adhesion and required for gamete activation depends on this protein kinase located in the cytoplasm.

\section{Discussion}

We screened for cell-cell fusion defects in several Chlamydomonas minus mating type strains from the CLiP mutant library annotated to have disruptions in gamete-specific protein kinase genes. Out of 9 strains, we identified one, with a mutation in the mixed lineage protein kinase gene GSPK, that underwent ciliary adhesion with plus gametes similarly to $W T$ minus gametes but was strongly impaired in cell-cell fusion. gspk mutants were rescued for gamete fusion by introduction of a transgene encoding an HA-tagged form of GSPK, GSPK-HA. Cell fractionation and immunoblotting showed that GSPK-HA was present in cell bodies, with little if any in cilia. Immunoblotting in combination with treatment of cell lysates with a phosphatase enzyme showed that GSPK-HA was basally phosphorylated in naive gametes and that the entire cellular complement of GSPK was additionally phosphorylated within 1 minute after plus and minus gametes were mixed together. GSPK-HA was not phosphorylated when gametes were activated with db-cAMP, and db-cAMP treatment rescued fusion when added to gspk plus and minus gametes, indicating that phosphorylation of GSPK was not mediated by CAMP, and that GSPK functions upstream of the large increase in cAMP that induces gamete activation.

One of the most surprising findings was that all of the GSPK was phosphorylated within 1 minute after the gametes were mixed together (Fig. 2). Ciliary adhesion and consequent phosphorylation events indeed were terminated at the times indicated, because the samples for the immunoblots were placed directly into SDS-PAGE sample buffer and immediately heated. This response to engagement of receptors in cilia is much slower than that of olfaction, and slightly faster than that reported for the 
somatostatin receptor 3 pathway and the Hh pathway. In olfaction, the rapid increase

334 in cAMP within the cilia of olfactory epithelial cells triggered by binding of odorants to their GPCRs leads to changes in plasma membrane potential detectable within 100 $\mathrm{ms}^{46}$, nearly 3 orders of magnitude more rapid than for the GSPK response.

In the somatostatin pathway, mobilization of cytoplasmic $\beta$-arrestin 2 into cilia was detected by immunofluorescence within 4 minutes after addition of somatostatin to cultured hippocampal neurons ${ }^{47}$, a response time of similar magnitude, but slower than the GSPK response. In the Hh pathway, increases in full-length forms of Gli transcription factors within cilia were detected by immunofluorescence within 5 minutes after addition of the $\mathrm{Hh}$ ligand to cells in culture ${ }^{48}$. In more recent reports, increases or decreases in ciliary levels of several other Hh pathway proteins, including soluble and transmembrane proteins, were detected $\sim 15$ minutes after addition of the Hh ligand ${ }^{49,50}$.

One important difference between the GSPK and $\beta$-arrestin 2 responses and the Hh pathway response is that the first two depend on a signal sent from the cilium to the cell body, whereas the initial Hh pathway responses do not require communication between the cilium and the cell body but occur entirely within the cilium ${ }^{16}$. In the $\mathrm{Hh}$ pathway, Smo and the full-length Gli proteins are thought to move into and out of the cilium constitutively ${ }^{48,51,52}$. Through multiple, complex, and still emerging mechanisms, Hh binding to Patched activates Smoothened in the cilium, leading to Smoothened retention and consequent intraciliary alterations of $\mathrm{Gli}$ properties ${ }^{12,52-55}$. The $\mathrm{Hh}-$ dependent changes in cell proliferation that are the ultimate outcome of Hh pathway activation occur relatively much later ${ }^{56}$.

Our results indicate that ciliary adhesion indeed generates a signal that is sent to the cell body to elicit the large increase in cellular cAMP. That signal leads to phosphorylation of GSPK, and GSPK is required for the cAMP increase; but the failure of db-cAMP to induce GSPK phosphorylation indicates that the signal from the cilia is not cAMP, as been earlier suggested ${ }^{24}$, and thus the signal remains unknown.

364 Similarly, the rapid movement of $\beta$-arrestin from the cytoplasm to the cilia upon 
activation of the somatostatin receptor 3 (SSTR3) is proposed to be a response to an undefined signal from the cilium ${ }^{47}$.

Another consideration also argues against the notion that cAMP from Chlamydomonas cilia triggers the responses in the cell body. In addition to their critical signaling role in sexual reproduction, the two cilia drive motility. Under the control of cues from light shining on the channelrhodopsin-containing eyespots in cells, the beating of the two cilia can be differentially controlled to allow the cells to swim toward or away from the light and find favorable environments for photosynthetic growth. Although photoreceptor currents that regulate calcium concentrations are the primary controller of motiity ${ }^{57}$, cAMP also plays a role ${ }^{58}$. Our results that GSPK responds to non-cAMP-mediated signals from the cilia provides a solution to the potential problem that changes in light intensity experienced by gametes would activate them for cell fusion in the absence of a partner.

Results from a Chlamydomonas mutant with a phenotype similar to the gspk mutant raise ideas about the nature of the undefined ciliary signal. Gametes of the imp-3 mutant which have a lesion in the PP2A3 phosphatase ${ }^{59}$, also undergo normal ciliary adhesion, but adhesion fails to increase cAMP and fusion is strongly impaired. The substrates for PP2A3 are unknown, but earlier work on imp-3 mutant gametes suggested that this phosphatase functioned in the cilia, not the cell body ${ }^{60}$. Consistent with this earlier observation, immunofluorescence studies showed that PP2A3 was enriched in the proximal part of the cilia, just distal to the transition zone and some was also localized in the cell body ${ }^{59}$. One scenario for adhesion-induced gamete activation would be that SAG1-SAD1 interactions somehow modify ciliary PP2A3, which then moves to the cytoplasm to carry out its function, which could include changing the phosphorylation state of proteins that regulate GSPK properties. Future experiments with gametes bearing combinations of WT and mutant forms of GSPK and PP2A3 should provide new insights into the nature of the signal transmitted from the cilia.

We should note that because of the rapid kinetics of the responses of Chlamydomonas gametes to ciliary adhesion, we cannot rule out the possibility that this signaling 
system is similar to those of cilium-based olfaction and vision and depends on changes

399 in membrane potential. Indeed, Chlamydomonas possesses a gene,

400 ADCY1/Cre06.g300500, that encodes an unusual chimeric protein with a predicted N-

401 terminal channel-like domain and a C-terminal adenylyl cyclase domain. Moreover,

402 our gamete transcriptome results ${ }^{43}$ showed that ADCY1 transcripts are gamete-

403 specific and upregulated during gamete activation. ADCY1 homologs are present in

404 ciliated protozoa where they regulate motility ${ }^{61,62}$.

406 A possible clue about mechanisms for movement of a signal from the cilium to the cell

407 body comes from earlier work on the role of IFT in ciliary signaling in Chlamydomonas.

408 Studies with gametes of the fla10 temperature-sensitive mutant of the anterograde IFT

409 motor kinesin-2 (FLA10) showed that Chlamydomonas gametes whose cilia were

410 transiently depleted of their IFT machinery exhibited a ciliary signaling phenotype

411 identical to the gspk phenotype ${ }^{33,34}$. At 45 minutes after fla10 gametes were

412 transferred to the non-permissive temperature, IFT components were depleted from

413 the cilia, but the cilia remained essentially full-length and were undiminished in their

414 ability to undergo ciliary adhesion. Importantly, though, ciliary adhesion failed to

415 induce the typical increase in cAMP and failed to induce gamete activation and cell

416 fusion. As with the gspk mutants, gamete activation was rescued by addition of $\mathrm{db}$ -

417 cAMP. Thus, the signal for GSPK responses could be carried by retrograde IFT from

418 the cilia to the cytoplasm. It will be interesting to determine whether IFT is also required

419 for the SSTR3 and other GPCR responses in vertebrates. Unfortunately, the inability

420 to conditionally deplete vertebrate cilia of their IFT machinery makes such experiments

421 challenging. Conventional mutations in IFT proteins block ciliogenesis and conditional

422 IFT mutants are only just becoming available ${ }^{63}$.

424 Notably, not only is the cilium-to-cytoplasm signal in the somatostatin pathway 425 undefined, but (with the exceptions of odorant receptors, rhodopsin, and smoothened), 426 the cellular and molecular mechanisms that link ligand binding by the multitude of other 427 vertebrate ciliary GPCRs to responses in the cytoplasm remain largely unknown. 428 Current models are that cAMP from the cilium diffuses into the cytoplasm to regulate 429 effectors in the cytoplasm. But, whether cilium generated-cAMP that diffuses into the 
cytoplasm indeed is the signal is uncertain, and the localization and trafficking of effectors are still in early stages of investigation.

433 Our findings now set the stage for learning more about mechanisms of receptormediated cilium-to-cytoplasm communication. It will be important to learn whether, as we expect, the adhesion-induced phosphorylation of GSPK is essential for its function during gamete activation, and whether its protein kinase activity is required to induce the increase in cellular cAMP through as yet unidentified adenylyl cyclases or phosphodiesterases. Perhaps of even more importance, though, will be to use this system to investigate the undefined signal transmitted from the cilium to the cytoplasm and the mechanism of its transport.

\section{Acknowledgments}

We are grateful to Dr. Caren Chang, University of Maryland, College Park, MD, USA for insightful discussions. We thank our laboratory colleagues, Drs. Jennifer Pinello and Jun Zhang for their constructive insights. We acknowledge the Imaging Core Facility in the department of Cell Biology and Molecular Genetics at the University of Maryland, College Park for Leica TCS SP5 confocal microscope. This work was supported by National Institutes of Health Grant GM122565 to W. J. S.

\section{Author Contributions}

452 Conceptualization: M.A., P.R., W.J.S. Investigation: M.A., P.R., W.J.S. Methodology: 453 M.A., P.R., P.H., S.K., W.J.S. Resources: M.A., P.R., P.H., S.K., W.J.S. Writing: , M.A., 454 P.R., S.K., W.J.S. Reviewing: M.A., P.R., S.K., P.H., W.J.S.

\section{Declaration of Interests}

457 The authors declare no competing interests. 


\section{Contact for Reagent and Resource Sharing}

464 Requests for further information or resources and reagents should be directed to and will be fulfilled by the Lead Contact, William J. Snell (wsnell1@umd.edu).

\section{Cells and cell culture}

468 Chlamydomonas reinhardtii wild type strains 21gr (mating type plus; mt+; CC-1690; 469 designated WT(+), CMJ030 (mating type minus; mt-; CC-5325; designated WT(-), 470 hap2 (40D4; CC5281) and SAG1-HA strains used in this study were grown in liquid 471 tris-acetate phosphate medium (TAP) medium containing trace metals) at $22^{\circ} \mathrm{C}$ with aeration, or on the TAP plates with $1.5 \%$ agar $^{36}$. The Chlamydomonas CLiP library mutants were obtained from the Chlamydomonas Resource Center. These mutants were generated by the insertion of a DNA cassette (CIB1) conferring resistance to paromomycin into the Chlamydomonas strain CMJ03064. Upon receipt, each mutant was streaked to single colonies, genomic DNA was isolated using Clontech plant genomic DNA isolation reagent (Takara, Cat. No. 9194), and the insertion site of the CIB1 cassette was verified by PCR. The PCR primers used to confirm the insertions are listed in Supplementary Table 1.

\section{Plasmid construction and transformation into Chlamydomonas}

483 To prepare a plasmid containing an HA-tagged GSPK gene, a gene fragment of $8780 / 8769 \mathrm{bp}$ that included the full-length GSPK gene sequence (7272 bp) and an additional $850 \mathrm{bp} 5$ ' to the annotated transcription start site predicted to include the endogenous promoter and an additional $647 \mathrm{bp} 3$ ' to the stop codon was amplified from DNA of BAC clone 34G21 by PCR using primers possessing Xho1 and Not1 restriction sites at the 5' and 3' ends, respectively. The amplified PCR product was cloned into a paromomycin resistance vector, pChlamiRNA3int (obtained from the

490 Chlamydomonas Resource Center) in between Xho1 and Not1 restriction sites by Infusion HD EcoDry cloning plus kit (Takara, Cat. No. 638915). A gene fragment encoding three copies of the 9-amino-acid HA epitope followed by EcoR1 and Xbal restriction sites was inserted using QuikChange II XL Site-Directed Mutagenesis Kit (Agilent technologies). The resulting GSPK-HA transgene plasmid (13,468 bp) 
Chlamydomonas transformation, $p G S P K-H A$ was linearized with $B s p H 1$ and the purified, linear plasmid was electroporated into $21 \mathrm{gr} \mathrm{mt}+$ and CC-5325 mtChlamydomonas strains ${ }^{65}$. Transformants that grew on TAP plates containing paromomycin (Sigma, Cat. \# P5057 ) were picked into 96-well plates and screened for the presence of GSPK-HA by PCR using primers listed in Supplementary Table 1. PCR-positive transformants were screened for GSPK-HA expression by immunoblotting with an anti-HA antibody. medium into $\mathrm{N}$-free medium followed by growth under continuous light with aeration or agitation overnight. For gamete activation experiments, plus and minus gametes were mixed together or gametes of single mating types were experimentally activated by incubation in $\mathrm{N}$-free medium containing $15 \mathrm{mM}$ db-cAMP and $150 \mu \mathrm{M}$ papaverine (db-cAMP buffer) for $\sim 10$ minutes or more ${ }^{22}$. Cell-cell adhesion was quantified using an electronic particle counter (Coulter, Palo Alto, CA) as described previously ${ }^{39,66}$. Assays for cell wall loss and gamete fusion were as described previously ${ }^{44,67}$.

\section{Cell fractionation, cilia isolation, and assaying PKG phosphorylation}

515 Fractionation of the cells into cell bodies and cilia from naive and adhering gametes was carried out as described earlier ${ }^{36}$. Phosphorylation of PKG was assayed in vitro as described earlier ${ }^{36}$ using a protein tyrosine kinase (PTK) assay. $20 \mu$ l of whole cilia ( $\sim 3 \mu \mathrm{g} / \mu \mathrm{l}$ protein) in $5 \%$ sucrose, $20 \mathrm{mM}$ HEPES buffer were mixed with $20 \mu \mathrm{l}$ of $2 \mathrm{X}$ PTK buffer (20 mM HEPES, pH 7.2, 10 mM MgCl2, 2 mM dithiothreitol, 1 mM EDTA, $50 \mathrm{mM} \mathrm{KCl}, 2 \mathrm{mM}$ ATP, $0.2 \%$ Nonidet P-40, $0.4 \mathrm{mM}$ orthovanadate, $20 \mathrm{mM} \beta-$ glycerolphosphate, and $2 \%$ Sigma plant protease inhibitor cocktail) in the presence of ATP for 10 minutes and the phosphorylated form of PKG was detected by use of 4$20 \%$ gradient SDS-PAGE gels and immunoblotting using anti-phospho-tyrosine (antip-Tyr) antibody (Sigma, Cat. \# 05-321).

527 GSPK phosphorylation in lysates of naive, adhering, or db-cAMP-activated gametes 
was asssed by changes in migration in immunoblots. The reactions were stopped by addition of aliquots to 4xSDS sample buffer followed by immediate boiling. For phosphatase treatment, the lysates was prepared by brief sonication of $2 \times 10^{7}$ cells $/ \mathrm{ml}$ in $1 \mathrm{ml}$ in HEMDK buffer. The $40 \mu \mathrm{l}$ final reaction volume contained $31 \mu \mathrm{l}$ cell lysate,

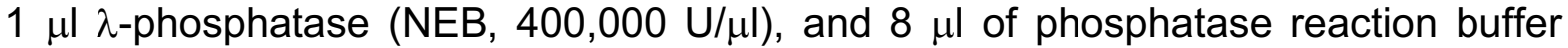
(NEB). After incubation at $30^{\circ} \mathrm{C}$ for 30 minutes, reactions were terminated by adding $40 \mu \mathrm{l}$ of $4 \times$ SDS sample buffer followed by boiling. As controls, samples were incubated in the presence of a phosphatase inhibitor cocktail (Sigma Cat. No. P2850).

\section{cAMP ELISA assay}

CAMP levels in adhering wildtype and GSPK mutant gametes were quantified by use of a cAMP Elisa kit (Enzo Life Sciences, \#ADI-900-163). Equal numbers (100 $\mu$ l, 2X10 ${ }^{7}$ cells $/ \mathrm{ml}$ in M-N) of the WT and gspk plus and minus gametes were separately mixed in $1.5 \mathrm{ml}$ Eppendorf tubes to initiate ciliary adhesion, and at the times indicated the cells were harvested by centrifugation $\left(6350 \times \mathrm{g} ; 4^{\circ} \mathrm{C}\right)$ and flash-frozen in liquid nitrogen. For the assay, samples were resuspended in $100 \mu \mathrm{l}$ of $0.1 \mathrm{M} \mathrm{HCl}$ and incubated at room temperature for 10 minutes followed by clarification by centrifugation $\left(20000 \times \mathrm{g} ; 4^{\circ} \mathrm{C}\right)$. Supernatants were transferred to fresh tubes for use in the assay, which was performed using the acetylation protocol according to the manufacturer's instructions. Absorbance at $405 \mathrm{~nm}$ of standards and experimental samples were determined using a microplate reader (LabSystems-Multiskan Ascent 354 Microplate Reader, San Diego, CA, USA). Results shown are from 6 independent experiments and are plotted as pmol/ml cAMP produced in the WT and gspk mutant mixtures.

\section{Determination of mating structure activation.}

554 As described previously ${ }^{68}$, samples ( 200 $\mu \mathrm{l}, 5 \times 10^{6}$ cells/ ml) in N-free medium were seeded on cover slips coated with poly-L-lysine (Sigma, Cat. No. P8920) for 5 minutes followed by fixation with $4 \%$ paraformaldehyde solution (Sigma, Cat. No. 158127) freshly made in $10 \mathrm{mM}$ HEPES, $\mathrm{pH}$ 7.4. Coverslips were washed with $1 \mathrm{x}$ PBS for 3 minutes, immersed for 6 minutes in 80\% acetone (Fischer Scientific, Cat. No. 67-64- 
560 immersion in $100 \%$ acetone at $-20^{\circ} \mathrm{C}$ for 6 minutes. Samples were then stained with 561 Allexa 488 Phalloidin (ThermoFisher Scientific, Cat. No. A12379) for 15 minutes in the 562 dark ${ }^{69}$. Phalloidin incubation was followed by a wash in 1x PBS for 5 minutes. Finally, 563 coverslips were mounted on slides using antifading agent Fluoromount-G ${ }^{\mathrm{TM}}$ 564 (ThermoFisher Scientific, Cat. No. 00-4959-02) and examined by Hyd detectorequipped Leica TCS SP5 confocal microscope using a 1.4 numerical aperture, $63 \mathrm{X}$ oil immersion objective. Images obtained from $z$ series were summed to produce a projected image using Leica LAS X, and cropped in the Illustrator program of Adobe Systems (USA).

\section{Protein Determination, SDS PAGE and Immunoblotting}

Protein concentrations were determined by use of the Bradford assay (Bio-Rad protein assay kit II, Cat. No. 5000002). For immunoblotting, samples were separated by SDSPAGE on 4-20\% Tris-Glycine or SDS-MOPS gradient gels (GenScript, USA) and transferred onto PVDF membranes (Merck Millipore, Cat. No. IPVH00010) as described previously ${ }^{34,40}$. Membranes were blocked by incubation in $3 \%$ fat-free dried milk (Carnation, Nestle, Inc., Solon, $\mathrm{OH}$ ) for 1 hour followed by 1 hour of incubation in the primary antibody. Membranes were washed three times for 10 minutes with TBST (Tris-buffered saline, $0.1 \%$ Tween 20 ) followed by incubation with secondary antibody. After three consecutive washes with TBST and incubation in the chemiluminescent substrate, fluorescence signals were captured on a C-Digit blot scanner (LI-COR Instruments, USA). The antibodies used for immunoblotting were rat anti-HA (1:3000; Roche), mouse anti-a-tubulin (1:5000; Sigma) and goat anti-rat IgG $\operatorname{HRP}(1: 5000$; Merck); and goat anti-mouse IgG HRP (1:5000; Sigma).

\section{Bioinformatic Analysis, Quantification and Statistical Analysis}

586 For comparative analysis the homologous protein sequences were aligned with

587 ClustalW, and the percentage of positions with identical or identical plus similar amino 588 acid positions were calculated using the BioEdit 7.2 software 589 (https://bioedit.software.informer.com/7.2/) software with a threshold of 75\%. JPred4 590 was used to predict secondary structure ${ }^{70}$. N-terminal myristoylation ( $\mathrm{N}$-myristoylation)

591 sites were predicted using NMT - The MYR Predictor 592 (http://mendel.imp.ac.at/myristate/SUPLpredictor.htm). All quantitative data represent 
593 at least three independent sets of experiments. Statistical significance of differences

594 between groups was assessed by Student's t-test. Data were analyzed using 595 GraphPad Prism 9 (GraphPad Software, U.S.A.).

\section{References:}

599

600

6011 Mykytyn, K. \& Askwith, C. G-Protein-Coupled Receptor Signaling in Cilia. Cold 602 Spring Harb Perspect Biol 9, doi:10.1101/cshperspect.a028183 (2017).

6032 Singla, V. \& Reiter, J. F. The primary cilium as the cell's antenna: signaling at 604 a sensory organelle. Science 313, 629-633, doi:10.1126/science.1124534 (2006).

6063 Hildebrandt, F., Benzing, T. \& Katsanis, N. Ciliopathies. N Engl J Med. 364, 1533-1543, doi:10.1056/NEJMra1010172 (2011).

6084 Dell'Orco, D., Koch, K. W. \& Rispoli, G. Where vision begins. Pflugers Arch 473, 1333-1337, doi:10.1007/s00424-021-02605-3 (2021).

Nakamura, T. Cellular and molecular constituents of olfactory sensation in vertebrates. Comp Biochem Physiol A Mol Integr Physiol 126, 17-32, doi:10.1016/s1095-6433(00)00191-4 (2000).

6136 Dwyer, N. D., Troemel, E. R., Sengupta, P. \& Bargmann, C. I. Odorant receptor localization to olfactory cilia is mediated by ODR-4, a novel membraneassociated protein. Cell 93, 455-466, doi:10.1016/s0092-8674(00)81173-3 (1998).

6177 Christensen, S. T., Morthorst, S. K., Mogensen, J. B. \& Pedersen, L. B. Primary Cilia and Coordination of Receptor Tyrosine Kinase (RTK) and Transforming Growth Factor beta (TGF-beta) Signaling. Cold Spring Harb Perspect Biol 9, doi:10.1101/cshperspect.a028167 (2017).

6218 Wu, C. T. et al. Discovery of ciliary G protein-coupled receptors regulating pancreatic islet insulin and glucagon secretion. Genes Dev, doi:10.1101/gad.348261.121 (2021).

6249 Huangfu, D. et al. Hedgehog signalling in the mouse requires intraflagellar transport proteins. Nature 426, 83-87, doi:10.1038/nature02061 (2003). 
62610 Pazour, G. J., Quarmby, L., Smith, A. O., Desai, P. B. \& Schmidts, M. Cilia in cystic kidney and other diseases. Cell Signal 69, 109519, doi:10.1016/j.cellsig.2019.109519 (2020).

62911 Ma, M. Cilia and polycystic kidney disease. Semin Cell Dev Biol 110, 139-148, doi:10.1016/j.semcdb.2020.05.003 (2021).

12 Nachury, M. V. The molecular machines that traffic signaling receptors into and out of cilia. Curr Opin Cell Biol 51, 124-131, doi:10.1016/j.ceb.2018.03.004 (2018).

63413 Garcia, G., 3rd, Raleigh, D. R. \& Reiter, J. F. How the Ciliary Membrane Is Organized Inside-Out to Communicate Outside-In. Curr Biol 28, R421-R434, doi:10.1016/j.cub.2018.03.010 (2018).

$14 \mathrm{Hu}, \mathrm{Q}$. et al. A septin diffusion barrier at the base of the primary cilium maintains ciliary membrane protein distribution. Science 329, 436-439, doi:10.1126/science.1191054 (2010).

64015 Dhallan, R. S., Yau, K. W., Schrader, K. A. \& Reed, R. R. Primary structure and functional expression of a cyclic nucleotide-activated channel from olfactory neurons. Nature 347, 184-187, doi:10.1038/347184a0 (1990).

16 Truong, M. E. et al. Vertebrate cells differentially interpret ciliary and extraciliary cAMP. Cell 184, 2911-2926 e2918, doi:10.1016/j.cell.2021.04.002 (2021).

17 Nachury, M. V. \& Mick, D. U. Establishing and regulating the composition of cilia for signal transduction. Nat Rev Mol Cell Biol 20, 389-405, doi:10.1038/s41580-019-0116-4 (2019). Adipogenesis. Cell 179, 1289-1305 e1221, doi:10.1016/j.cell.2019.11.005 (2019).

19 Saada, E. A. et al. Insect stage-specific receptor adenylate cyclases are localized to distinct subdomains of the Trypanosoma brucei Flagellar membrane. Eukaryot Cell 13, 1064-1076, doi:10.1128/EC.00019-14 (2014). in African Trypanosomes. mBio 6, e01954-01914, doi:10.1128/mBio.01954-14 (2015). 
21 Kawano, M., Tominaga, T., Ishida, M. \& Hori, M. Roles of Adenylate Cyclases in Ciliary Responses of Paramecium to Mechanical Stimulation. J Eukaryot Microbiol 67, 532-540, doi:10.1111/jeu.12800 (2020).

660

Pasquale, S. M. \& Goodenough, U. W. Cyclic AMP functions as a primary sexual signal in gametes of Chlamydomonas reinhardtii. The Journal of cell biology 105, 2279-2292 (1987).

23 Pijst, H. L. A., van Driel, R., Janssens, P. M. W., Musgrave, A. \& van den Ende, $\mathrm{H}$. Cyclic AMP is involved in sexual reproduction of Chlamydomonas eugametos. FEBS Letters 174, 132-136, doi:10.1016/0014-5793(84)81091-1 (1984).

Saito, T., Small, L. \& Goodenough, U. W. Activation of adenylyl cyclase in Chlamydomonas reinhardtii by adhesion and by heat. J Cell Biol 122, 137-147, doi:10.1083/jcb.122.1.137 (1993). Zhang, Y. H., Ross, E. M. \& Snell, W. J. ATP-dependent regulation of flagellar adenylyl cyclase in gametes of Chlamydomonas reinhardtii. J Biol Chem 266, 22954-22959 (1991).

Zhang, Y. \& Snell, W. J. Flagellar adhesion-dependent regulation of Chlamydomonas adenylyl cyclase in vitro: a possible role for protein kinases in sexual signaling. J Cell Biol 125, 617-624, doi:10.1083/jcb.125.3.617 (1994).

Adair, W. S., Hwang, C. \& Goodenough, U. W. Identification and visualization of the sexual agglutinin from the mating-type plus flagellar membrane of Chlamydomonas. Cell 33, 183-193, doi:10.1016/0092-8674(83)90347-1 (1983).

28 Ferris, P. J. et al. Plus and minus sexual agglutinins from Chlamydomonas reinhardtii. Plant Cell 17, 597-615, doi:10.1105/tpc.104.028035 (2005).

29 Zhang, Y. \& Snell, W. J. Differential regulation of adenylyl cyclases in vegetative and gametic flagella of Chlamydomonas. J Biol Chem 268, 1786-1791 (1993). Ranjan, P., Awasthi, M. \& Snell, W. J. Transient Internalization and Microtubule-Dependent Trafficking of a Ciliary Signaling Receptor from the Plasma Membrane to the Cilium. Curr Biol 29, 2942-2947 e2942, doi:10.1016/j.cub.2019.07.022 (2019). 
Musgrave, A. et al. Evidence for a functional membrane barrier in the transition zone between the flagellum and cell body of Chlamydomonas eugametos gametes. Planta 167, 544-553 (1986). Hunnicutt, G. R., Kosfiszer, M. G. \& Snell, W. J. Cell body and flagellar agglutinins in Chlamydomonas reinhardtii: the cell body plasma membrane is a reservoir for agglutinins whose migration to the flagella is regulated by a functional barrier. J Cell Biol 111, 1605-1616, doi:10.1083/jcb.111.4.1605 (1990).

33 Pan, J. \& Snell, W. J. Kinesin-II is required for flagellar sensory transduction during fertilization in Chlamydomonas. Mol Biol Cell 13, 1417-1426, doi:10.1091/mbc.01-11-0531 (2002). membrane protein entry into flagella is facilitated by cytoplasmic microtubules does doi:10.1016/j.cub.2013.06.025 (2013).

Lechtreck, K. F. et al. Cycling of the signaling protein phospholipase D through

cilia requires the BBSome only for the export phase. J Cell Biol 201, 249-261, doi:10.1083/jcb.201207139 (2013).

Wang, Q. \& Snell, W. J. Flagellar adhesion between mating type plus and mating type minus gametes activates a flagellar protein-tyrosine kinase during fertilization in Chlamydomonas. J Biol Chem 278, 32936-32942, doi:10.1074/jbc.M303261200 (2003).

Wang, Q., Pan, J. \& Snell, W. J. Intraflagellar Transport Particles Participate Directly in Cilium-Generated Signaling in Chlamydomonas. Cell 125, 549-562, doi:10.1016/j.cell.2006.02.044 (2006). Solter, K. M. \& Gibor, A. Evidence for role of flagella as sensory transducers in mating of Chlamydomonas reinhardtii. Nature 265, 444-445, doi:10.1038/265444a0 (1977).

9 Snell, W. J. \& Moore, W. S. Aggregation-dependent turnover of flagellar adhesion molecules in Chlamydomonas gametes. J Cell Biol 84, 203-210, doi:10.1083/jcb.84.1.203 (1980). 
40 Cao, M. et al. Uni-directional ciliary membrane protein trafficking by a cytoplasmic retrograde IFT motor and ciliary ectosome shedding. Elife 4:e05242, doi:10.7554/eLife.05242 (2015).

41 Goodenough, U. W. Cyclic AMP enhances the sexual agglutinability of Chlamydomonas flagella. J Cell Biol 109, 247-252, doi:10.1083/jcb.109.1.247 (1989).

42 Goodenough, U. W. \& Weiss, R. L. Interrelationships between microtubules, a striated fiber, and the gametic mating structure of Chlamydomonas reinhardtii. J Cell Biol 76, 430-438 (1978).

43 Ning, J. et al. Comparative genomics in Chlamydomonas and Plasmodium identifies an ancient nuclear envelope protein family essential for sexual reproduction in protists, fungi, plants, and vertebrates. Genes Dev 27, 11981215, doi:10.1101/gad.212746.112 (2013).

Liu, Y. et al. The conserved plant sterility gene HAP2 functions after attachment of fusogenic membranes in Chlamydomonas and Plasmodium gametes. Genes Dev 22, 1051-1068, doi:10.1101/gad.1656508 (2008).

45 Saito, T., Tsubo, Y. \& Matsuda, Y. Synthesis and turnover of cell body agglutinin as a pool of flagellar surface agglutinin in Chlamydomonas reinhardtii gamete. Archives of Microbiology 142, $207-210$ (1985).

Paoli, M. et al. Neuronal Response Latencies Encode First Odor Identity Information across Subjects. J Neurosci 38, 9240-9251, doi:10.1523/JNEUROSCI.0453-18.2018 (2018).

47 Green, J. A. et al. Recruitment of beta-Arrestin into Neuronal Cilia Modulates 235, doi:10.1128/MCB.00765-15 (2016).

Wen, X. et al. Kinetics of hedgehog-dependent full-length Gli3 accumulation in primary cilia and subsequent degradation. Mol Cell Biol 30, 1910-1922, doi:10.1128/MCB.01089-09 (2010).

49 Pal, K. et al. Smoothened determines beta-arrestin-mediated removal of the G protein-coupled receptor Gpr161 from the primary cilium. J Cell Biol 212, 861875, doi:10.1083/jcb.201506132 (2016). response. J Cell Biol 220, doi:10.1083/jcb.202007207 (2021). 
75251 Ye, F. et al. Single molecule imaging reveals a major role for diffusion in the exploration of ciliary space by signaling receptors. Elife 2, e00654, doi:10.7554/eLife.00654 (2013).

Milenkovic, L., Scott, M. P. \& Rohatgi, R. Lateral transport of Smoothened from the plasma membrane to the membrane of the cilium. J Cell Biol 187, 365-374, doi:10.1083/jcb.200907126 (2009).

53 Petrov, K., Wierbowski, B. M., Liu, J. \& Salic, A. Distinct Cation Gradients Power Cholesterol Transport at Different Key Points in the Hedgehog Signaling Pathway. Dev Cell 55, 314-327 e317, doi:10.1016/j.devcel.2020.08.002 (2020).

54 Rohatgi, R., Milenkovic, L. \& Scott, M. P. Patched1 regulates hedgehog signaling at the primary cilium. Science 317, 372-376, doi:10.1126/science.1139740 (2007).

55 Zhang, Y. et al. Structural Basis for Cholesterol Transport-like Activity of the Hedgehog Receptor Patched. Cell 175, 1352-1364 e1314, doi:10.1016/j.cell.2018.10.026 (2018).

Arveseth, C. D. et al. Smoothened transduces Hedgehog signals via activitydependent sequestration of PKA catalytic subunits. Plos Biol 19, e3001191, doi:10.1371/journal.pbio.3001191 (2021).

57 Harz, H. \& Hegemann, P. Rhodopsin-regulated calcium currents in Chlamydomonas. Nature 351, 489-491, doi:doi.org/10.1038/351489a0 (1991).

58 Saegusa, Y. \& Yoshimura, K. cAMP controls the balance of the propulsive forces generated by the two flagella of Chlamydomonas. Cytoskeleton (Hoboken) 72, 412-421, doi:10.1002/cm.21235 (2015). identifies a deletion in protein phosphatase $2 \mathrm{~A}$ that affects its stability and localization in Chlamydomonas reinhardtii. PLoS Genet 9, e1003841, doi:10.1371/journal.pgen.1003841 (2013). doi:10.1126/science.164.3882.963 (1969). 
62 Weber, J. H. et al. Adenylyl cyclases from Plasmodium, Paramecium and Tetrahymena are novel ion channel/enzyme fusion proteins. Cell Signal 16, 115-125, doi:10.1016/s0898-6568(03)00129-3 (2004).

63 Engelke, M. F. et al. Acute Inhibition of Heterotrimeric Kinesin-2 Function Reveals Mechanisms of Intraflagellar Transport in Mammalian Cilia. Curr Biol 29, 1137-1148 e1134, doi:10.1016/j.cub.2019.02.043 (2019).

$\mathrm{Li}, \mathrm{X}$. et al. A genome-wide algal mutant library and functional screen identifies genes required for eukaryotic photosynthesis. Nature Genetics 51, 627-635, doi:10.1038/s41588-019-0370-6 (2019).

65 Shimogawara, K., Fujiwara, S., Grossman, A. \& Usuda, H. High-efficiency transformation of Chlamydomonas reinhardtii by electroporation. Genetics 148 , 1821-1828 (1998).

66 Snell, W. J. \& Roseman, S. Kinetics of adhesion and de-adhesion of Chlamydomonas gametes. J Biol Chem 254, 10820-10829 (1979).

67 Snell, W. J. Study of the release of cell wall degrading enzymes during adhesion of Chlamydomonas gametes. Exp Cell Res 138, 109-119, doi:10.1016/0014-4827(82)90096-9 (1982).

Wilson, N. F., Foglesong, M. J. \& Snell, W. J. The Chlamydomonas mating type plus fertilization tubule, a prototypic cell fusion organelle: isolation, characterization, and in vitro adhesion to mating type minus gametes. J Cell Biol 137, 1537-1553, doi:10.1083/jcb.137.7.1537 (1997).

69 Craig, E. W. et al. The elusive actin cytoskeleton of a green alga expressing both conventional and divergent actins. Mol Biol Cell 30, 2827-2837, doi:10.1091/mbc.E19-03-0141 (2019).

70 Drozdetskiy, A., Cole, C., Procter, J. \& Barton, G. J. JPred4: a protein secondary structure prediction server. Nucleic Acids Res 43, W389-394, doi:10.1093/nar/gkv332 (2015). 


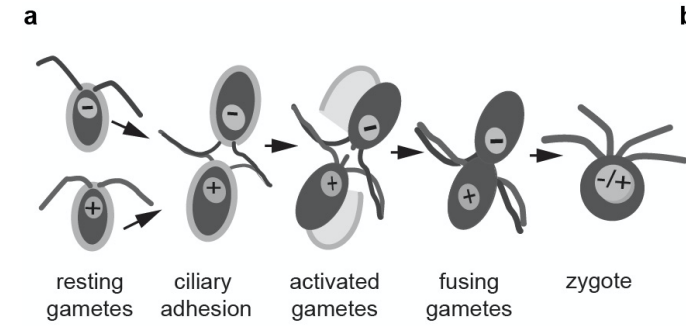

c

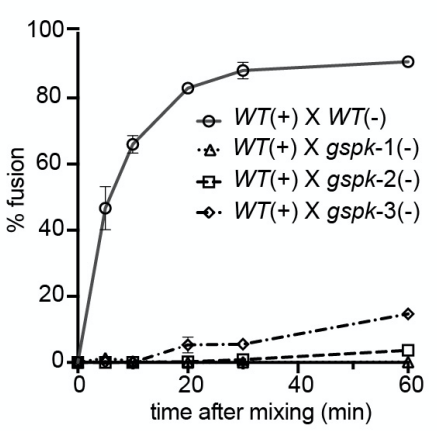

d

LMJ.RY0402.138658 (gspk-1)
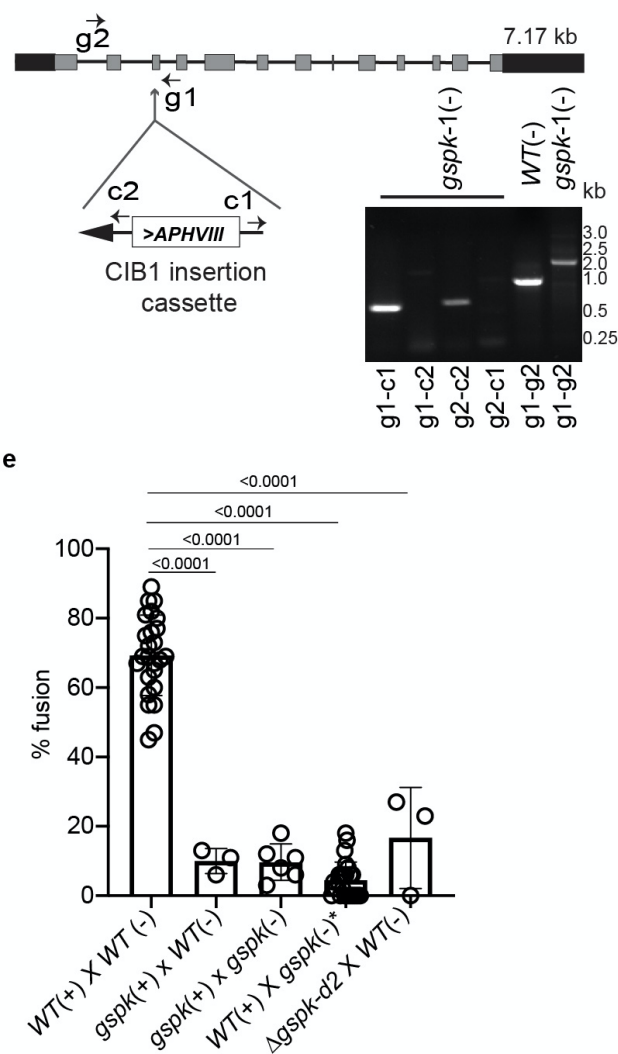

\begin{tabular}{|c|c|c|c|c|c|c|c|}
\hline \multirow[b]{2}{*}{ Protein } & \multirow[b]{2}{*}{ Gene ID } & \multicolumn{5}{|c|}{$\begin{array}{c}\text { Transcript abundance } \\
\text { (RPKM) }\end{array}$} & \multirow{2}{*}{$\begin{array}{l}\text { Fusion } \\
\text { phenotype } \\
\text { of mutant }\end{array}$} \\
\hline & & V & mt- $G$ & $\begin{array}{c}\mathrm{mt}-\mathrm{G} \\
\mathrm{db}-\mathrm{cAMP}\end{array}$ & $\mathrm{mt}+\mathrm{G}$ & $\begin{array}{c}m t+G \\
\text { db-cAMP }\end{array}$ & \\
\hline $\begin{array}{l}\text { RPL19- } \\
\text { SAG1 - } \\
\text { SAD1 }\end{array}$ & $\begin{array}{r}\text { - Cre02.g075700 } \\
\text { - Cre08.g374250 } \\
\text { - Cre06.g255000 } \\
\text { Cre12.g537400 }\end{array}$ & $\begin{array}{c}1694 \\
0 \\
0 \\
2\end{array}$ & $\begin{array}{c}1551 \\
1 \\
28 \\
77\end{array}$ & $\begin{array}{c}1907 \\
0 \\
168 \\
626\end{array}$ & $\begin{array}{l}1491 \\
79 \\
0 \\
229\end{array}$ & $\begin{array}{c}1536 \\
344 \\
0 \\
681\end{array}$ & $\begin{array}{l} \\
\text { no fusion } \\
\text { no fusion } \\
\text { wt }\end{array}$ \\
\hline 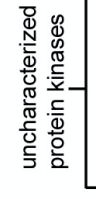 & $\begin{array}{l}\text { Cre02.g107000 } \\
\text { Cre02.g12500 } \\
\text { Cre06.g287450 } \\
\text { Cre12.g510600 } \\
\text { Cre02.g087750 } \\
\text { Cre16.g689300 } \\
\text { Cre10.g435750 } \\
\text { Cre02.g104450 }\end{array}$ & $\begin{array}{l}2 \\
2 \\
1 \\
1 \\
2 \\
3 \\
3 \\
3 \\
0\end{array}$ & $\begin{array}{c}48 \\
73 \\
16 \\
13 \\
15 \\
17 \\
0 \\
10\end{array}$ & $\begin{array}{l}0 \angle 0 \\
188 \\
10 \\
30 \\
74 \\
37 \\
3 \\
0 \\
0\end{array}$ & $\begin{array}{l}229 \\
75 \\
60 \\
8 \\
23 \\
19 \\
18 \\
5 \\
24\end{array}$ & $\begin{array}{c}601 \\
81 \\
6 \\
6 \\
38 \\
5 \\
1 \\
1 \\
2\end{array}$ & $\begin{array}{l}w \\
w t \\
w t \\
w t \\
w t \\
w t \\
w t \\
w t \\
\text { impaired fusio }\end{array}$ \\
\hline
\end{tabular}

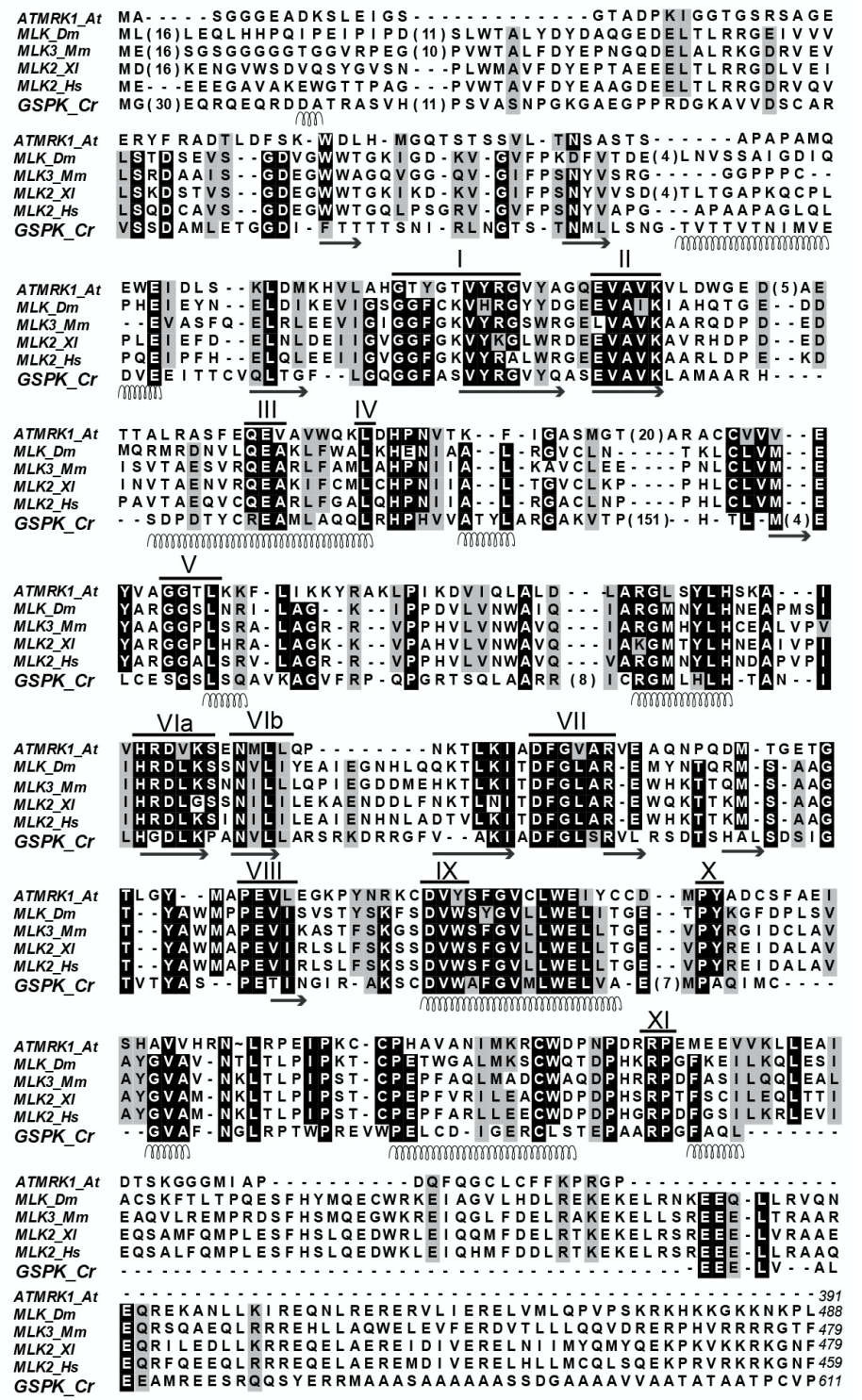

Figure 1. GSPK is a gamete-specific mixed lineage protein kinase essential for gamete fusion. a Illustration of fertilization in Chlamydomonas. b The Cre02.g104450 
820 vegetatative $(V)$, naive minus (mt- $\mathrm{G}$ ) and plus $(\mathrm{mt}+\mathrm{G})$ gametes, and db-cAMP821 activated minus (mt- G, db-cAMP) and plus (mt+ G, db-cAMP) gametes represented 822 by median reads per kilobase per million mapped reads (RPKM) are from Ning et al. 823 (2013). RPL19 is a housekeeping gene. The right panel indicates the fusion 824 phenotypes. c Fusion is impaired in multiple Cre02.g104450 mutant strains. 825 Quantification of fusion of $W T(+)$ gametes with minus gametes of $W T$ and three 826 different CLiP gspk mutants. Values are means (+/- standard deviation) from three 827 replicates. d Structure of the GSPK genomic locus in the gspk-1 CLiP mutant. Grey 828 solid boxes indicate exons; thin lines, introns; UTRs are shown as black boxes. The 829 CIB1 (AphVIII) insertion cassette is in the 3rd intron of gspk in the gspk allele of 830 LMJ.RY0402.138658_1 strain. Primer locations for genotyping are shown by arrows, 831 where g1 and g2 are gene-specific primers and c1 and c2 are specific to the insertion 832 cassette. Gel images show diagnostic genomic DNA PCR samples. Primer 833 combinations used for PCR are indicated at the bottom of the lanes. e Quantification 834 of fusion in WT gametes ( $\mathrm{n}=25$ experiments) compared with fusion in $g s p k(+)$ mutant progeny generated from a cross between $g s p k(-)$ and $W T(+)(n=3)$; fusion of $g s p k(+)$

836 mutants mixed with $g s p k(-)(n=7)$; fusion of $g s p k^{*}(-)$ mutant progeny generated from a 837 cross between $g s p k(+)$ and $W T(-)(n=25)$; and fusion of CRISPR-generated $\Delta g s p k-$ $838 d 2(+)$ mutant mixed with $W T(-)(n=3)$. P-values labelled above the groups compared 839 are from Student's t-test. (F) Multiple sequence alignment of GSPK and protein 840 kinases from Arabidopsis thaliana (BAA22079.1), Drosophila melanogaster 841 (AAL08011.1), Mus musculus (AAF73281.1), Xenopus leavis (AAP46399.1), and 842 Homo sapiens (CAA62351.1). Identical and similar residues are highlighted in black 843 and grey, respectively. The conserved domains of the catalytic core are indicated by 844 roman numerals. Regions with $\alpha$-helices (spirals) and $\beta$-sheets (arrows) predicted for 845 GSPK by JPred4 are denoted below the sequences. 


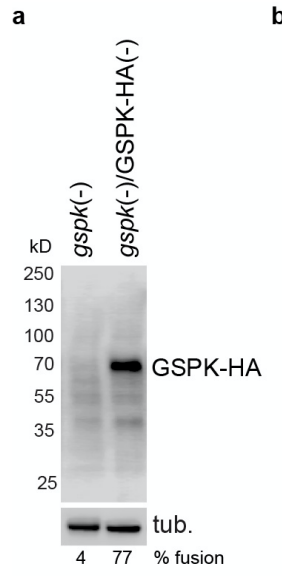

d

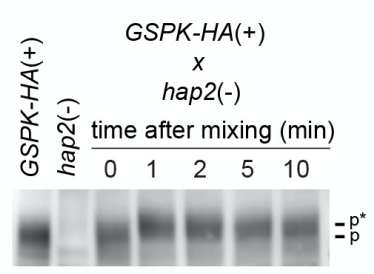

f
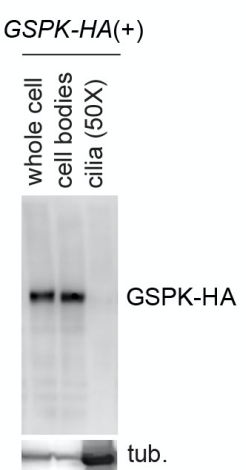

g

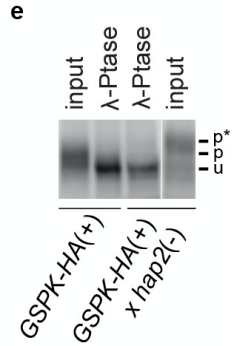

850 Figure 2: GSPK is gamete-specific and phosphorylated within 1 minute after ciliary adhesion is initiated. a Expression of GSPK-HA in gspk minus gametes rescues fusion. Anti-HA immunoblot of gspk(-) and GSPK-HA-rescued gspk(-) gametes. Percent fusion at 10 minutes is shown below the blot. Tubulin was used as

854 a loading control. b GSPK-HA expression is gamete-specific and reduced upon 855 gamete activation by db-cAMP. Anti-HA immunoblot of $g s p k / G S P K-H A(+)$ vegetative 856 cells $(V)$, naive gametes $(\mathrm{G})$, and gametes activated by incubation in db-cAMP buffer 857 (G-A). c GSPK-HA is basally phosphorylated in naive gametes. Anti-HA immunoblot 858 of lysates of $g s p k / G S P K-H A(+)$ naive gametes that had been incubated with $\lambda$ phosphatase in the presence and absence of a phosphatase inhibitor. d-e GSPK-HA 
860 is phosphorylated within 1 minute after initiation of ciliary adhesion. Anti-HA 861 immunoblots of GSPK-HA(+) gametes at the indicated times after mixing with hap2 (-) 862 gametes (d). Anti-HA immunoblot of lysates of gspk/GSPK-HA(+) gametes before and 86310 minutes after mixing with hap2(-) gametes with and without treatment with $\lambda$ 864 phosphatase (e). Letters on the right indicate GSPK-HA that is unphosphorylated $(u)$, 865 basally phosphorylated $(p)$, or additionally phosphorylated $\left(p^{*}\right)$. f GSPK fractionates with cell bodies. Anti-HA immunoblot of whole cells, cell bodies, and cilia of gspk/GSPK-HA(+) gametes. $3 \mu \mathrm{g}$ of protein were loaded per lane, which for cilia represents about 50 cell equivalents. The lower panel is a tubulin loading control. $\mathbf{g}$ Activation of gametes with db-cAMP buffer fails to induce phosphorylation of GSPKHA. Anti-HA immunoblot of gspk/GSPK-HA(+) gametes at the indicated times after mixing with db-cAMP buffer. Cell wall loss was over $80 \%$ at 10 minutes, confirming gamete activation.
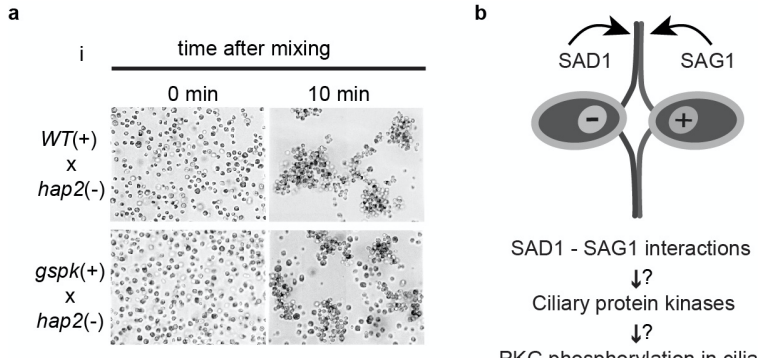

SAD1 - SAG1 interactions
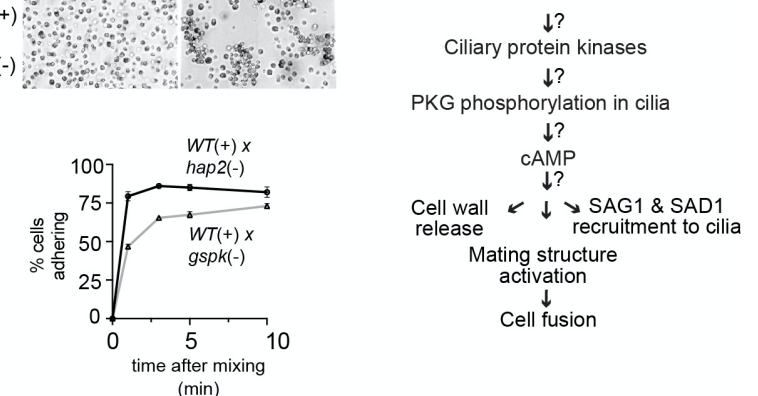

Mating structure
activation

Cell fusion

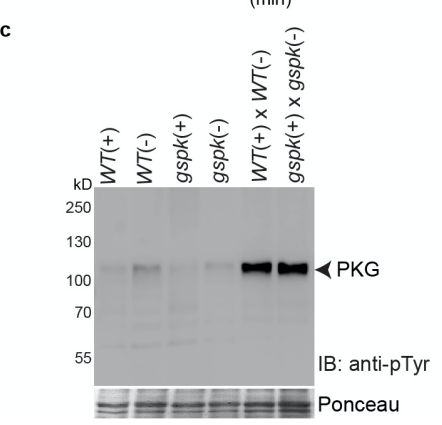

876 Figure 3: gspk mutants undergo ciliary adhesion and phosphorylation of PKG 
Differential Interference Contrast (DIC) micrographs of $W T(+)$ gametes (upper panel) and $g s p k(+)$ gametes (lower panel) at the times indicated after mixing with hap2(-) gametes. aii Quantification of adhesion in the indicated samples by use of an electronic particle counter. b Schematic diagram of cilium-generated signaling 882 pathway in Chlamydomonas. c Ciliary adhesion in gspk gametes induces 883 phosphorylation of cGMP-dependent protein kinase (PKG). Anti-p-Tyr immunoblots 884 from PTK assay of cilia isolated from non-adhering WT(+), WT(-), gspk(+) and gspk(-) gametes and from $W T(+)$ gametes mixed with $W T(-)$ gametes and gspk(+) gametes mixed with gspk(-) gametes.

887

888

a

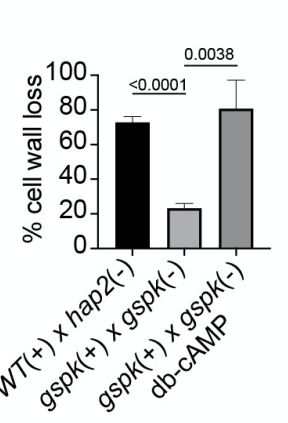

b
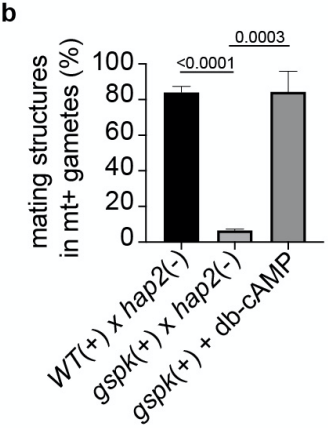

C

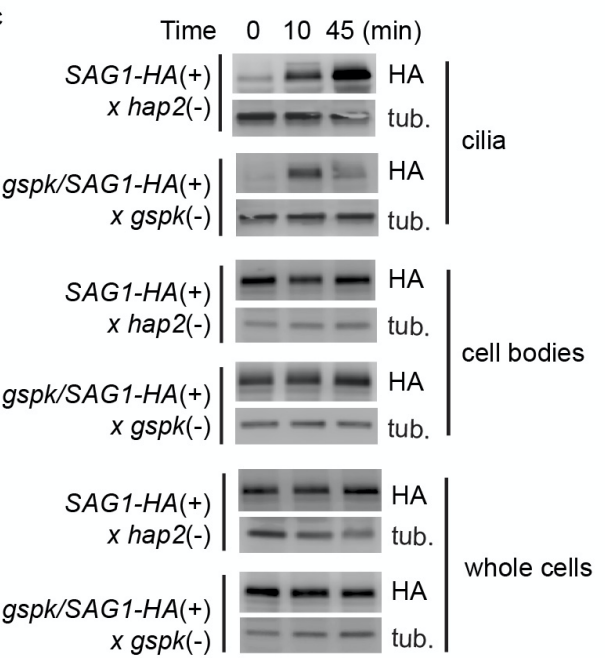

d
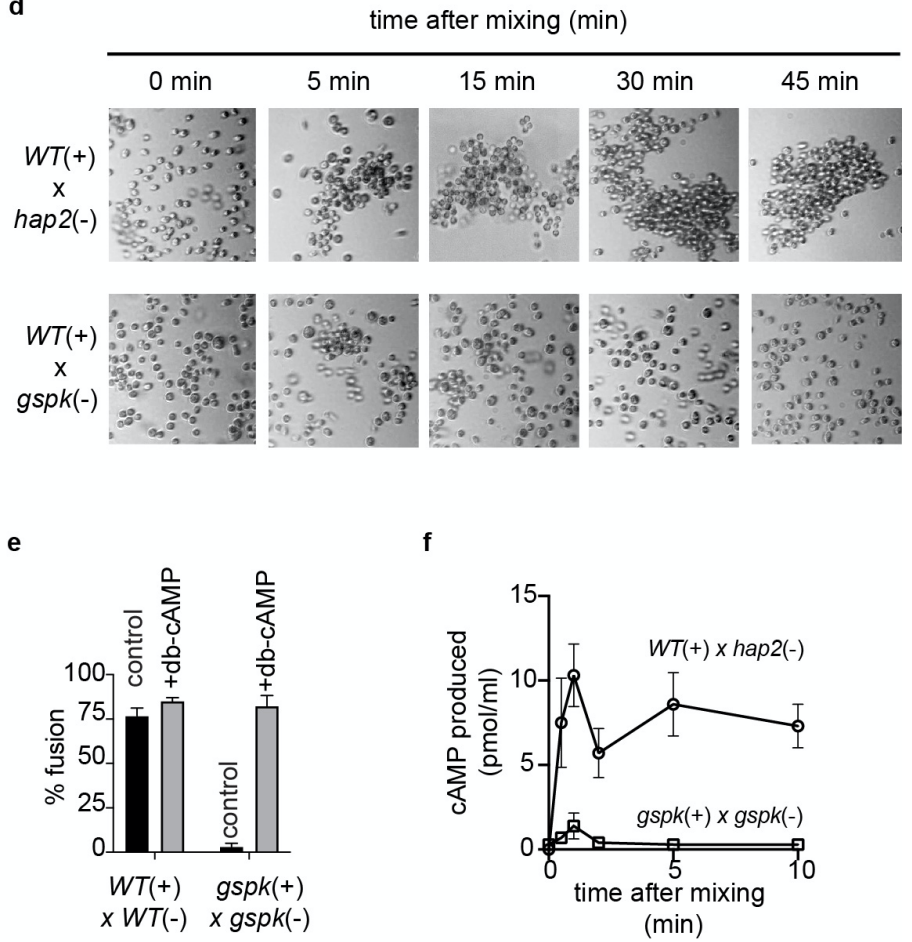

889

Figure 4: Ciliary adhesion-induced cell body responses are impaired in gspk mutants and restored by treatment with db-cAMP, and ciliary adhesion fails to 
895 wall loss and mating structure activation. Quantification of cell wall loss at 10 minutes 896 after mixing $W T(+)$ and hap2(-) gametes and $g s p k(+)$ and $g s p k(-)$ gametes in the 897 absence and presence of db-cAMP buffer (a). Quantification of mating structure 898 formation in mixtures of WT(+) and hap2(-) gametes and gspk(+) and gspk(-) gametes 899 at 10 minutes after mixing and in samples of $g s p k(+)$ gametes that had been incubated 900 with db-cAMP buffer for 30 minutes (b). c Ciliary adhesion-induced movement of 901 SAG1-HA from the cell plasma membrane to the ciliary membrane is impaired in 902 gspk(+) gametes. Anti-HA immunoblots of whole cells, cell bodies, and cilia from the 903 indicated samples harvested 0,10 and 45 minutes after SAG1-HA-expressing WT and $904 g s p k(+)$ gametes were separately mixed with gspk(-) gametes. The lower panel is a 905 tubulin loading control. $3 \mu \mathrm{g}$ protein were loaded in each lane. $\mathbf{d}$ gspk gametes fail to 906 undergo sustained ciliary adhesion. Bright field micrographs of samples taken at the 907 indicated times after mixing $W T(+)$ gametes with hap2(-) gametes and WT(+) gametes 908 with gspk(-) gametes. e Fusion in gspk gametes is rescued by db-cAMP. 909 Quantification of fusion in mixtures of WT plus and minus gametes and gspk plus and 910 minus gametes treated with or without db-cAMP. f The ciliary adhesion-induced 911 increase in cAMP is impaired in gspk gametes. cAMP concentrations were determined 912 by use of an ELISA-based method at the indicated times after mixing wild-type plus 913 and minus gametes and gspk plus and minus gametes. P-values shown for $A$ and $B$ 914 are from comparisons of means by Student's t-test.

915

\section{Supplementary information}

\begin{tabular}{cccc}
\cline { 2 - 4 } Primers & LMJ.RY0402.138658 & LMJ.RY0402.097798 & LMJ.RY0402.039382 \\
\cline { 2 - 4 } g1 & GCACATAAGGTAGGGCGTGT & GTAAATCAAGCTCCCTGCCA & ACCAACAGGAGAATATGGCG \\
g2 & TTGCTTATATGCTTGCGTGC & GCTGCCTCATTACCTCTTGC & GGGTGATGTCATTAATCGGG \\
c1 & GCACCAATCATGTCAAGCCT & GCACCAATCATGTCAAGCCT & GCACCAATCATGTCAAGCCT \\
C2 & GACGTTACAGCACACCCTTG & GACGTTACAGCACACCCTTG & GACGTTACAGCACACCCTTG
\end{tabular}

920 Supplementary Table 1: AphVII cassette and gene-specific primers used for CLiP 921 mutant analysis. 

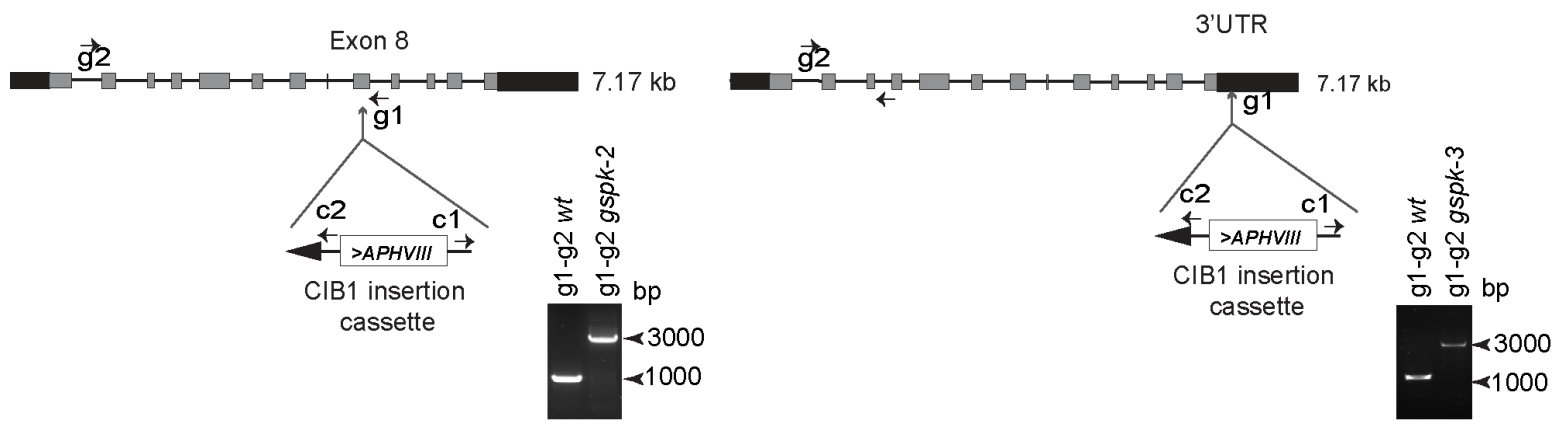

Supplementary Fig. 1: Structure of the GSPK genomic locus in CLiP library mutants.

a-b. Grey solid boxes indicate exons; thin lines indicate introns; UTRs are shown as black boxes. The CIB1 (AphVIII) insertion cassette is located in the 8th intron of gspk in the gspk allele of LMJ.RY0402.097798_1 strain; gspk-2 (a) and in the 3'UTR of gspk in the gspk allele of LMJ.RY0402.039382_1 strain; gspk-3 (b). Primer locations for genotyping are shown by arrows where g1 and g2 are gene-specific primers and c1 and c2 are the primers specific to the insertion cassette. Gel images show the diagnostic genomic DNA PCR gels. The primer combinations used for PCR are document the AphVIII insertions.

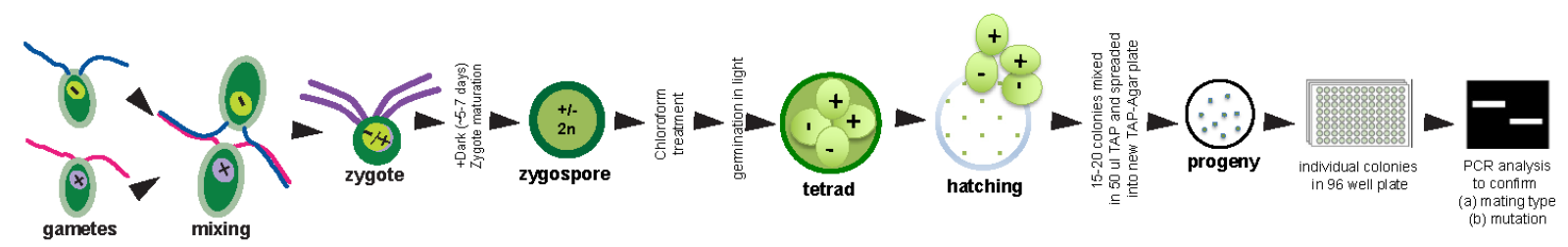

Supplementary Fig. 2: Graphical overview of method for obtaining progeny of desired genotypes from crosses of Chlamydomonas gametes. 
bioRxiv preprint doi: https://doi.org/10.1101/2021.09.03.458889; this version posted September 4, 2021. The copyright holder for this preprint (which was not certified by peer review) is the author/funder, who has granted bioRxiv a license to display the preprint in perpetuity. It is made available under aCC-BY-NC-ND 4.0 International license.

a

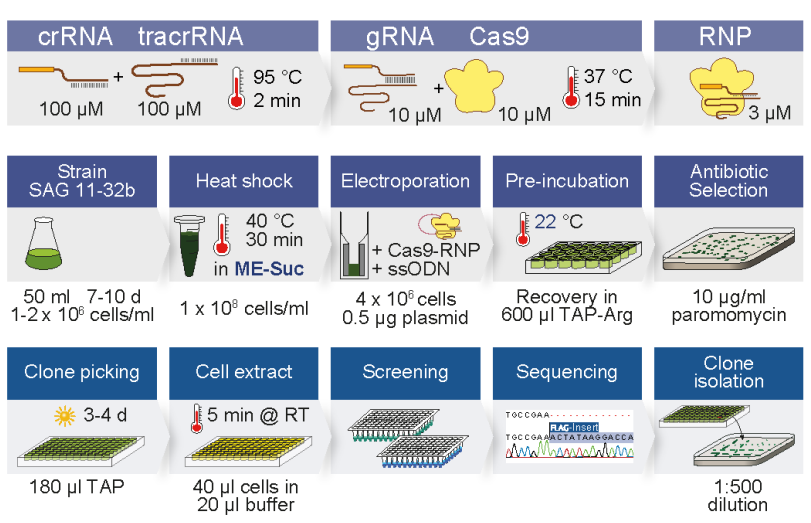

\section{b}

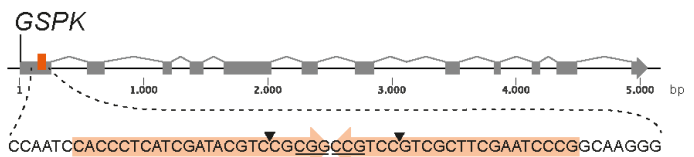
gRNA GSPK-b gRNA GSPK-a (r139)

(r138)

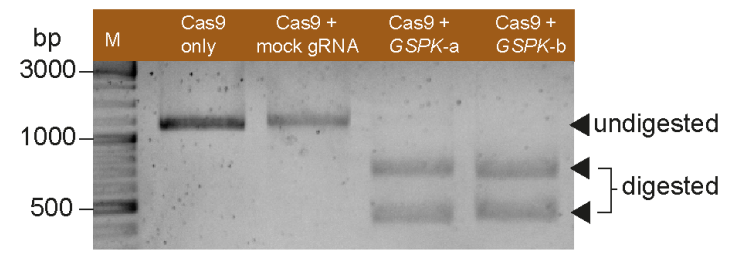

C

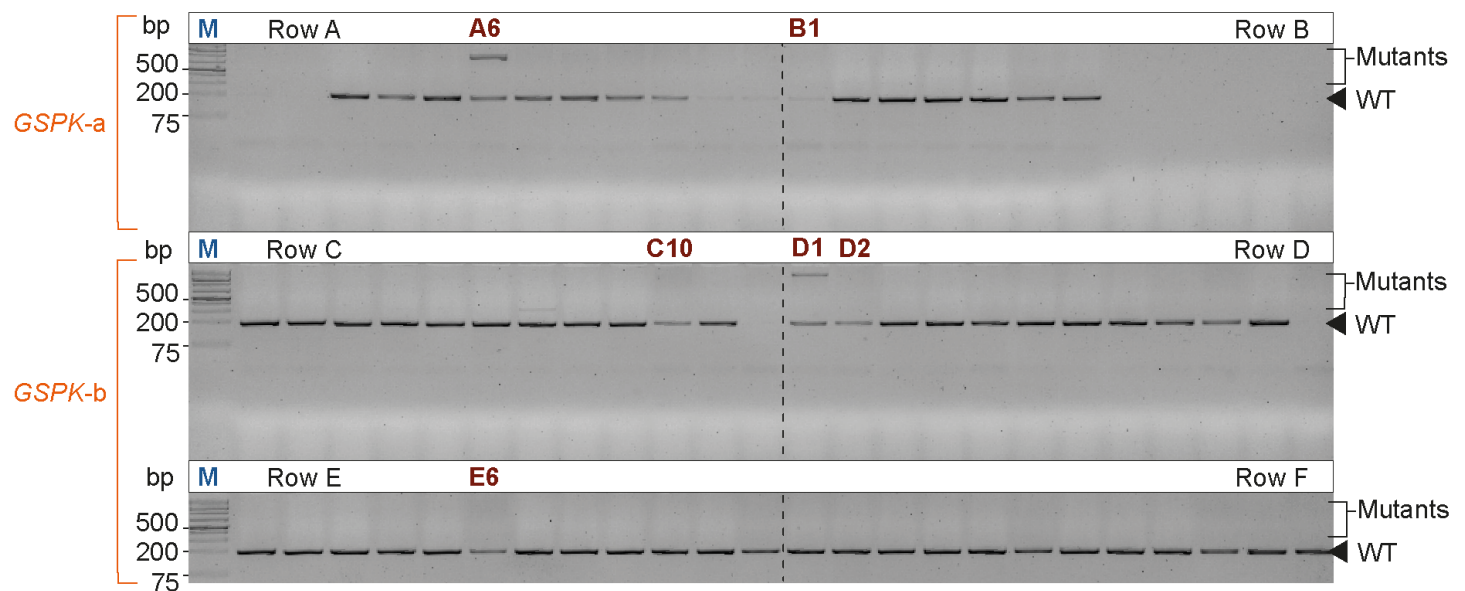

d

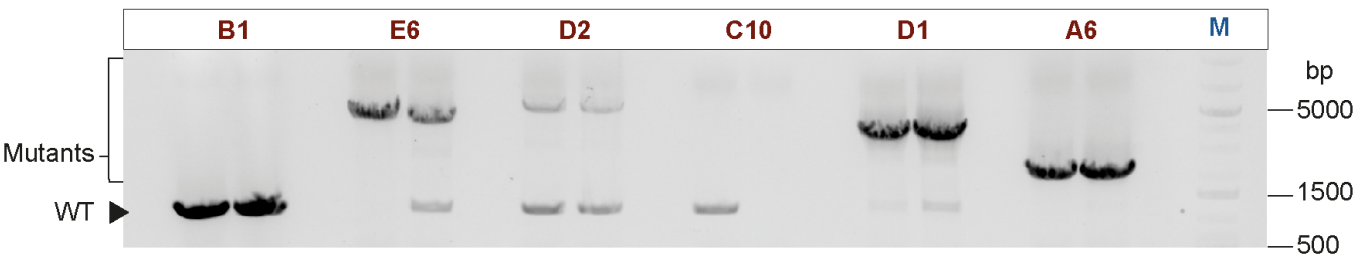

e

GSPK-b

WT GSPK GTGCACGGTGCCAATCCACCCTCATCGATACGT....

CCGGCGGCCGTCCGTCGCTTCGAATCCCGGCAAGGG

agspk-d1 GTGCACGAACCCCCCGTTCAGCCCGACCGCTGCGCCTIATCCGGTARCTATCGTCTIGAGTCCAACCCGGTAAG. / /ATTTGAGCACTTGCAACCCTTATCCGGAAGCCGCGGCCGTCCGTCGCTTCGAMTCCCGGCAAGGG

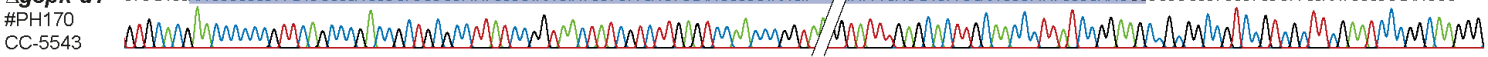
sgspk-d2 GTGCACGGTGCCAATCCACCCTCCACAGAATCAGGGGATAACGCAGGAAAGAACATGIGAGCAAAAGGCCAGCA / /AAAGCGGAGGAGIITIGCAATITGITGGTCCGGGGCCGTCCGTCGCTTCGAATCCGGGCAAGGG

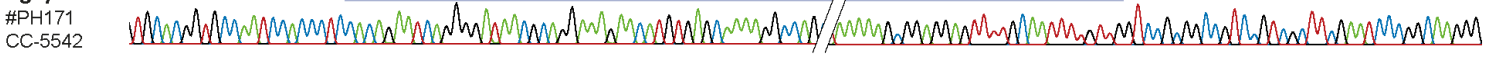


952 Supplementary Fig. 3: Generation of $\Delta g s p k-d 2$ CRISPR mutant.

953 a. Graphical overview of the methodological steps for the generation of a GSPK 954 mutant with CRISPR-Cas9 ribonucleoproteins (Cas9-RNPs). Taken and adapted from 955 (Kelterborn, 2020)

956 b. GSPK gene locus with two Cas9 target sites selected with the CRISPOR algorithm 957 (Concordet and Haeussler, 2018). Efficiency of the two selected guide RNAs (gRNAs) 958 was tested by in-vitro digestion as described in (Kelterborn, 2020). A PCR product 959 spanning both target sites was incubated with only Cas9 protein, with Cas9-RNPs 960 using a non-targeting mock gRNA, or with Cas9-RNPs assembled with GSPK-a or 961 GSPK-b gRNA. The two lower DNA bands indicate effective Cas9-induced cleavage 962 at the GSPK-a and GSPK-b target site.

963 c. Single colonies transformed with GSPK-a or GSPK-b Cas9-RNPs were analyzed 964 with a short colony-PCR spanning 197 bp containing both target sites. PCR bands 965 with a different size (clone A6, C7 and D1) or different intensity than WT bands (clones 966 A1, A2, A11, A12, C10, C12, D2, D10, E6) potentially indicate a mutated GSPK locus.

967 d. Clone A6, B1, C10, D1, D2, E6 were selected for further analysis with a larger locus 968 PCR (1157 bp) and using longer elongation times (3 min). PCR analysis reveal PCR 969 bands with WT size (B1), large insertions (A6, D1, D2 and E6) or missing PCR bands 970 (C10). A lower PCR band ( 1100 bp) can be seen in E6, D2, C10 and D1, and 971 potentially derives from a mixture of mutant and WT cells.

972 e. Clone $\Delta g s p k-d 1$ and $\Delta g s p k-d 2$ were singled out to remove remaining WT cells and 973 the mutation in the GSPK locus was confirmed by sequencing analysis. Both clones 974 show large insertions of the pAPHVIII marker plasmid leading to a premature stop 975 codon and consequently to disrupted GSPK gene expression. Cell-cell fusion results are shown for clone $\Delta g s p k-d 2$ CRISPR mutant. 
bioRxiv preprint doi: https://doi.org/10.1101/2021.09.03.458889; this version posted September 4, 2021. The copyright holder for this preprint (which was not certified by peer review) is the author/funder, who has granted bioRxiv a license to display the preprint in perpetuity. It is made available under aCC-BY-NC-ND 4.0 International license.

$W T(+)$
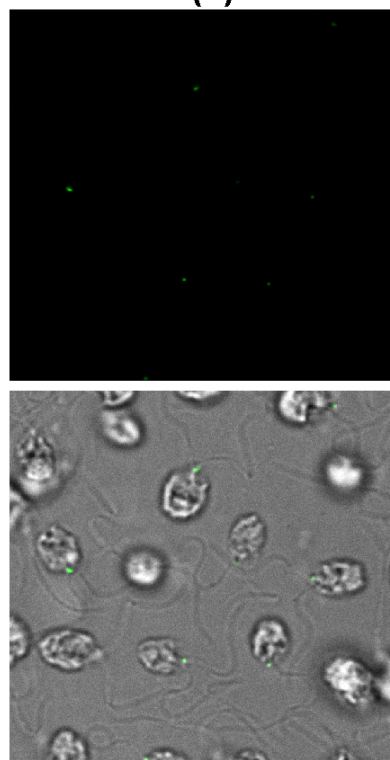

$W T(+) \times$ hap2(-)
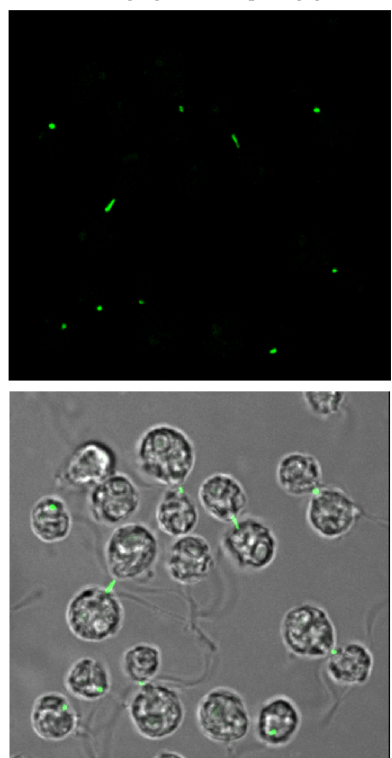

$W T+\mathrm{db}-\mathrm{cAMP}$
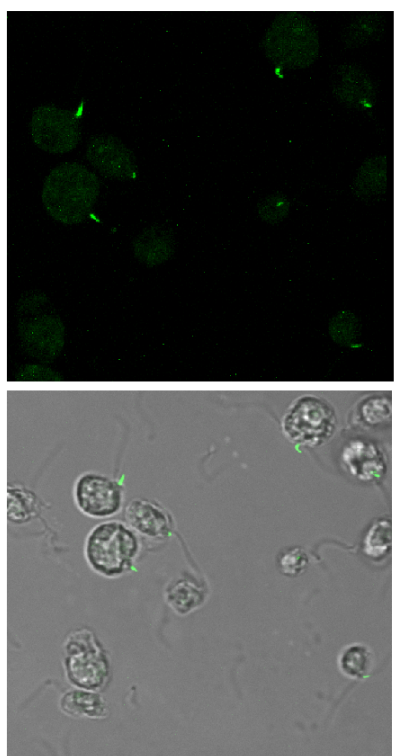

gspk(+)

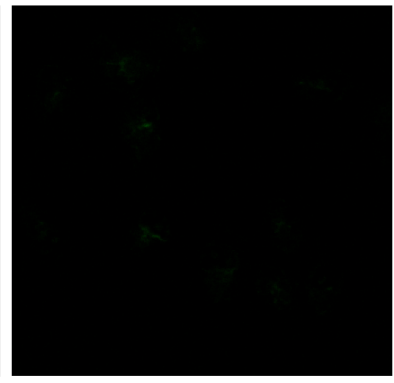

(9)

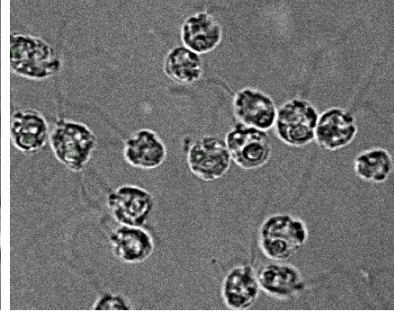

$\operatorname{gspk}(+) \times$ hap2(-)

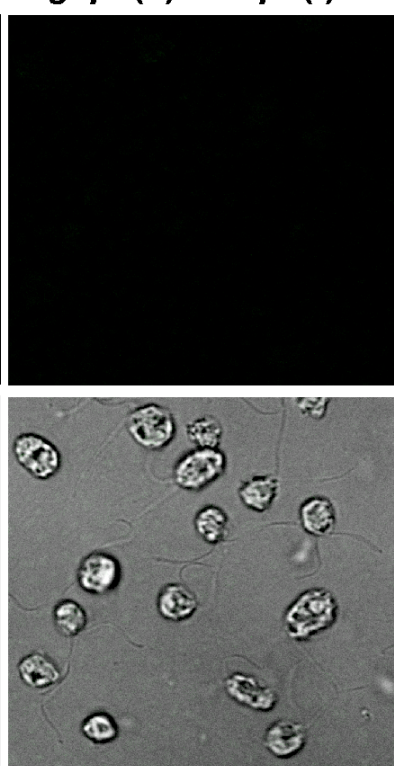

gspk(+) + db-cAMP
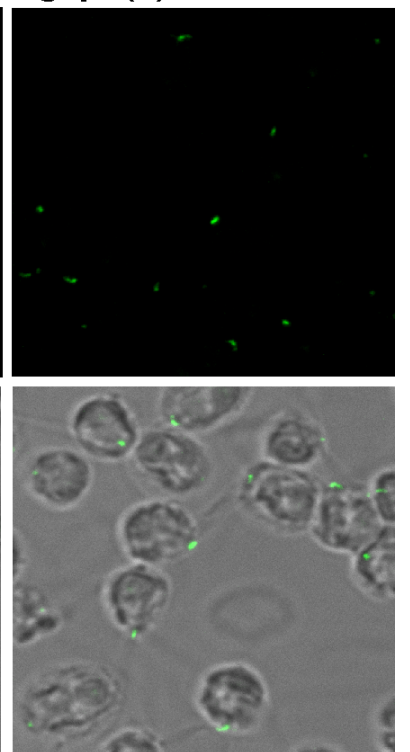
979 Supplementary Fig. 4: Ciliary adhesion induces formation of actin-filled fertilization

980 tubules in $W T(+)$ gametes but not in gspk plus gametes. Fluorescence images of cells

981 stained with Alexa 488 phalloidin showed fertilization tubules in mixtures of $W T(+)$ and

982 hap2(-) gametes, but not in mixtures of $g s p k(+)$ and hap2(-) gametes (upper panel).

983 Unmixed $W T(+)$ and $g s p k(+)$ gametes lack fertilization tubules (control, middle panel).

984 gspk(+) gametes activated with db-cAMP buffer formed fertilization tubules similarly

985 to $W T(+)$ gametes (lower panel).

986

987 Supplementary References:

988 Concordet, J.P., and Haeussler, M. (2018). CRISPOR: intuitive guide selection for

989 CRISPR/Cas9 genome editing experiments and screens. Nucleic Acids Res 46, 990 W242-W245.

991 Kelterborn, S. (2020). Gen-Editierung von Photorezeptorgenen in der Grünalge 992 Chlamydomonas reinhardtii mithilfe des CRISPR/Cas9-Systems. (Humboldt-

993 Universität zu Berlin).

994 